\title{
A MULTILAYER SAINT-VENANT SYSTEM WITH MASS EXCHANGES FOR SHALLOW WATER FLOWS. DERIVATION AND NUMERICAL VALIDATION*
}

\author{
Emmanuel Audusse ${ }^{1}$, Marie-Odile Bristeau ${ }^{2}$, Benoît Perthame ${ }^{2,3}$ \\ AND JACQUES SAINTE-MARIE ${ }^{2,4}$
}

\begin{abstract}
The standard multilayer Saint-Venant system consists in introducing fluid layers that are advected by the interfacial velocities. As a consequence there is no mass exchanges between these layers and each layer is described by its height and its average velocity. Here we introduce another multilayer system with mass exchanges between the neighboring layers where the unknowns are a total height of water and an average velocity per layer. We derive it from Navier-Stokes system with an hydrostatic pressure and prove energy and hyperbolicity properties of the model. We also give a kinetic interpretation leading to effective numerical schemes with positivity and energy properties. Numerical tests show the versatility of the approach and its ability to compute recirculation cases with wind forcing.
\end{abstract}

Mathematics Subject Classification. 35Q30, 35Q35, 76D05.

Received January 23, 2009. Revised December 15, 2009.

Published online June 24, 2010.

\section{INTRODUCTION}

Due to computational issues associated with the free surface Navier-Stokes or Euler equations, the simulations of geophysical flows are often carried out with shallow water type models of reduced complexity. Indeed, for vertically averaged models such as the Saint-Venant system [7], efficient and robust numerical techniques (relaxation schemes [10], kinetic schemes [24], ...) are available and avoid to deal with moving meshes.

Non-linear shallow water equations model the dynamics of a shallow, rotating layer of homogeneous incompressible fluid and are typically used to describe vertically averaged flows in two or three dimensional domains, in terms of horizontal velocity and depth variation, see Figure 1.

\footnotetext{
Keywords and phrases. Navier-Stokes equations, Saint-Venant equations, free surface, multilayer system, kinetic scheme.

* This work has been achieved while the authors were involved in the ANR project METHODE (http://methode.netcipia. net).

${ }^{1}$ Univ. Paris 13, Institut Galilée, 99 avenue Jean-Baptiste Clément, 93430 Villetaneuse, France. audusse@math. univ-paris13.fr

2 INRIA Paris-Rocquencourt, B.P. 105, 78153 Le Chesnay Cedex, France. Marie-Odile.Bristeau@inria.fr

3 Lab. J.-L. Lions, Univ. P. et M. Curie, BC187, 4 place Jussieu, 75252 Paris Cedex 05, France. benoit.perthame@upmc.fr

4 Saint-Venant Laboratory, 6 quai Watier, 78400 Chatou, France. Jacques.Sainte-Marie@inria.fr
} 


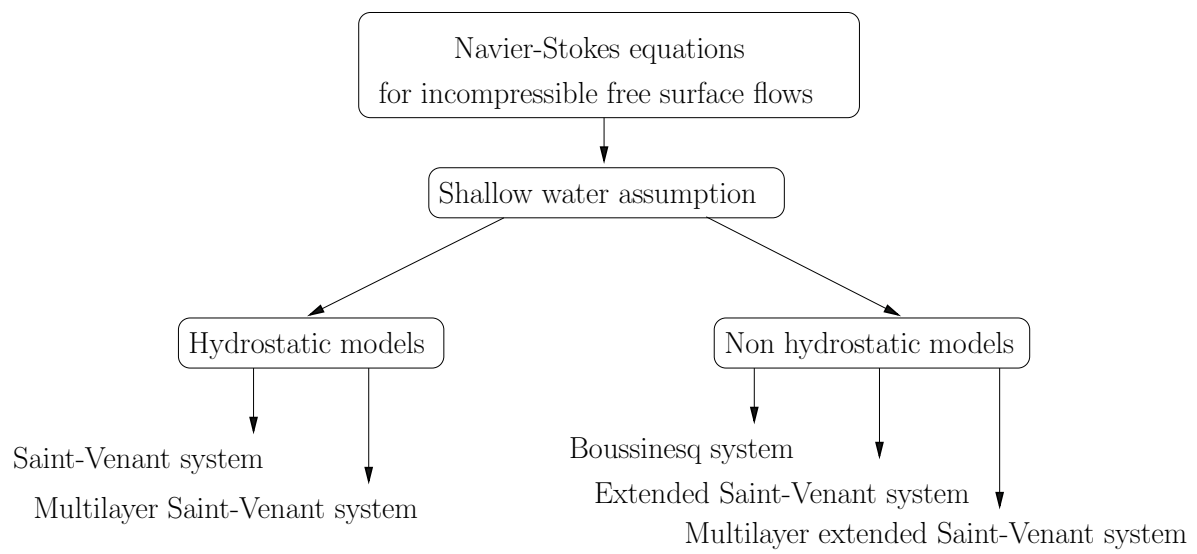

FiguRE 1. Averaged models derived from Navier-Stokes equations.

The classical Saint-Venant system [7] with viscosity and friction $[15,16,18,20]$ is well suited for the modeling of dam breaks or hydraulic jumps. The extended version of the Saint-Venant system proposed by Bristeau and Sainte-Marie [11] dropping the hydrostatic assumption is well adapted for the modeling of gravity waves propagation.

Considering flows with large friction coefficients, with significant water depth or with important wind effects, the horizontal velocity can hardly be approximated - as in the Saint-Venant system - by a vertically constant velocity [26]. To drop this limitation a multilayer Saint-Venant model is often used where each layer is described by its own height, its own velocity and is advected by the flow (see $[1,4,6]$ and the references therein). This advection property induces that there is no mass exchanges between neighboring layers and makes a close relation to models for two non-miscible fluids (see $[9,12,13]$ for instance). In [1], introducing a vertical partition of water height, a multilayer strategy was formally derived from the $2 \mathrm{~d}$ Navier-Stokes system with hydrostatic hypothesis and it is extended to $3 \mathrm{~d}$ computations in [6].

Here, we derive another and simpler multilayer model where we prescribe the vertical discretization of the layers taking into account the (unknown) total height of water. Using a Galerkin approximation in Lagrangian formulation, we obtain a system where the only additional unknowns are the layers velocities. This leads to a global continuity equation and allows mass exchanges between layers.

The objective of the paper is to present the derivation of this new multilayer model and to exhibit its main properties (hyperbolicity, energy equality, ...). Some simulations performed with a kinetic scheme [3] are presented at the end of the paper where we demonstrate the accuracy and robustness of the model in-between the Navier-Stokes and Saint-Venant systems.

The paper is organized as follows. In Section 2, we first present, in a simplified case, the formulation of the new multilayer Saint-Venant system starting from the hydrostatic Euler equations. In Section 3, we recall the Navier-Stokes system with a free moving boundary and its closure, and the shallow water system. We also introduce the multilayer formulation in the context of the hydrostatic assumption. In Section 4 we examine the main properties of the multilayer system and present a kinetic interpretation of the proposed model. This kinetic formulation leads to a numerical scheme detailed in Section 5 where some numerical validations are also shown.

\section{A SIMPLIFIED CASE}

Before deriving the complete version of the multilayer system, we illustrate the approach in a simple situation. Moreover this case emphasizes the main differences with the multilayer system proposed by Audusse [1]. 


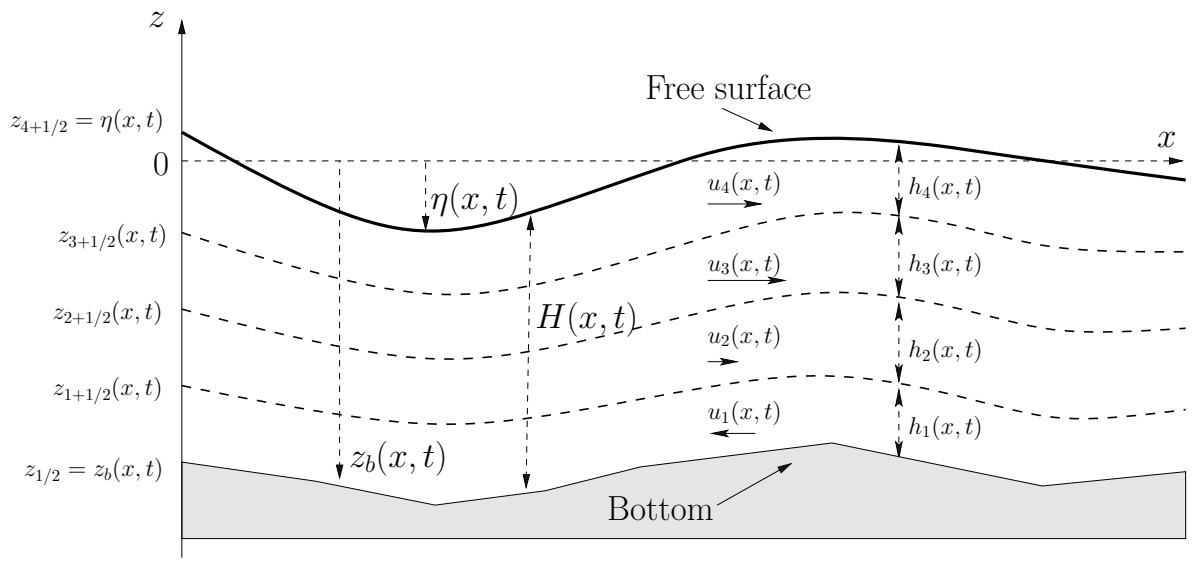

Figure 2. Notations for the multilayer approach.

We depart from the free surface hydrostatic Euler system

$$
\begin{aligned}
\frac{\partial u}{\partial x}+\frac{\partial w}{\partial z} & =0 \\
\frac{\partial u}{\partial t}+\frac{\partial u^{2}}{\partial x}+\frac{\partial u w}{\partial z}+\frac{\partial p}{\partial x} & =0 \\
\frac{\partial p}{\partial z} & =-g,
\end{aligned}
$$

for

$$
t>t_{0}, \quad x \in \mathbb{R}, \quad z_{b}(x) \leq z \leq \eta(x, t),
$$

where $\eta(x, t)$ represents the free surface elevation, $\mathbf{u}=(u, w)^{T}$ the velocity. The water height is $H=\eta-z_{b}$, see Figure 2 .

We add the two classical kinematic boundary conditions. At the free surface, we prescribe

$$
\frac{\partial \eta}{\partial t}+u_{s} \frac{\partial \eta}{\partial x}-w_{s}=0
$$

where the subscript $s$ denotes the value of the considered quantity at the free surface. At the bottom, the impermeability condition gives

$$
u_{b} \frac{\partial z_{b}}{\partial x}-w_{b}=0
$$

where the subscript $b$ denotes the value of the considered quantity at the bottom.

We consider that the flow domain is divided in the vertical direction into $N$ layers of thickness $h_{\alpha}$ with $N+1$ interfaces $z_{\alpha+1 / 2}(x, t), \alpha=0, \ldots, N$ (see Fig. 2) so that

$$
H=\sum_{\alpha=1}^{N} h_{\alpha},
$$

and

$$
z_{\alpha+\frac{1}{2}}(x, t)=z_{b}(x)+\sum_{j=1}^{\alpha} h_{j}(x, t) .
$$


We consider the average velocities $u_{\alpha}, \alpha=1, \ldots, N$ defined by

$$
u_{\alpha}(x, t)=\frac{1}{h_{\alpha}} \int_{z_{\alpha-1 / 2}}^{z_{\alpha}+1 / 2} u(x, z, t) \mathrm{d} z
$$

we also denote

$$
\left\langle u^{2}\right\rangle_{\alpha}(x, t)=\frac{1}{h_{\alpha}} \int_{z_{\alpha-1 / 2}}^{z_{\alpha}+1 / 2} u^{2}(x, z, t) \mathrm{d} z
$$

and

$$
u_{\alpha+1 / 2}=u\left(x, z_{\alpha+1 / 2}, t\right)
$$

the value of the velocity at the interface $z_{\alpha+1 / 2}$.

Proposition 2.1. With these notations, an integration of (2.1)-(2.3) over the layers $\left[z_{\alpha-1 / 2}, z_{\alpha+1 / 2}\right]$, $\alpha=1, \ldots, N$ leads to the following system of balance laws

$$
\begin{aligned}
\frac{\partial h_{\alpha}}{\partial t}+\frac{\partial h_{\alpha} u_{\alpha}}{\partial x} & =G_{\alpha+1 / 2}-G_{\alpha-1 / 2}, \\
\frac{\partial h_{\alpha} u_{\alpha}}{\partial t}+\frac{\partial}{\partial x}\left(h_{\alpha}\left\langle u^{2}\right\rangle_{\alpha}\right)+g h_{\alpha} \frac{\partial H}{\partial x} & =-g h_{\alpha} \frac{\partial z_{b}}{\partial x}+u_{\alpha+1 / 2} G_{\alpha+1 / 2}-u_{\alpha-1 / 2} G_{\alpha-1 / 2} .
\end{aligned}
$$

The expression of the exchange terms $G_{\alpha+1 / 2}$ is given in the following.

Proof. The proof relies on simple calculus based on the Leibniz rule. Using the incompressibility condition (2.1) integrated over the interval $\left[z_{\alpha-1 / 2}, z_{\alpha+1 / 2}\right]$, we deduce the mass equation (2.11) where we exhibit the kinematic of the interface on the right hand side

$$
G_{\alpha+1 / 2}=\frac{\partial z_{\alpha+1 / 2}}{\partial t}+u_{\alpha+1 / 2} \frac{\partial z_{\alpha+1 / 2}}{\partial x}-w\left(x, z_{\alpha+1 / 2}, t\right), \quad \alpha=0, \ldots, N .
$$

The relation (2.13) gives the mass flux leaving/entering the layer $\alpha$ through the interface $z_{\alpha+1 / 2}$.

Then we consider the velocity equation (2.2). We first observe that from the hydrostatic assumption (2.3) one can compute the pressure as a function of the water height :

$$
p(x, z, t)=g(\eta(x, t)-z) .
$$

Now we integrate equation (2.2) over the interval $\left[z_{\alpha-1 / 2}, z_{\alpha+1 / 2}\right]$ and we obtain the relation

$$
\frac{\partial h_{\alpha} u_{\alpha}}{\partial t}+\frac{\partial}{\partial x}\left(h_{\alpha}\left\langle u^{2}\right\rangle_{\alpha}\right)+g h_{\alpha} \frac{\partial \eta}{\partial x}=u_{\alpha+1 / 2} G_{\alpha+1 / 2}-u_{\alpha-1 / 2} G_{\alpha-1 / 2},
$$

and with the definition of $H$, this is equivalent to (2.12). Then the kinematic boundary conditions (2.4) and (2.5) can be written

$$
G_{1 / 2}=0, \quad G_{N+1 / 2}=0 .
$$

These equations just express that there is no loss/supply of mass through the bottom and the free surface.

Notice also that one can compute $G_{\alpha+1 / 2}$, just adding up the equations (2.11) for $j \leq \alpha$ and using the first equality of (2.15)

$$
G_{\alpha+1 / 2}=\frac{\partial}{\partial t} \sum_{j=1}^{\alpha} h_{j}+\frac{\partial}{\partial x} \sum_{j=1}^{\alpha} h_{j} u_{j}, \quad \alpha=1, \ldots, N
$$


The standard multilayer Saint-Venant system [1] is obtained by prescribing

$$
G_{\alpha+1 / 2} \equiv 0
$$

This choice is clearly natural for inmiscible fluids but is not justified if the multilayer system is seen as a numerical approximation of the hydrostatic Euler equations. Indeed there is no reason to prevent the water exchanges between connected layers. Moreover it is exhibited in [1] that this choice may lead to the development of instabilities at the interfaces.

Here we drop this assumption and we only keep the two physical kinematic boundary conditions (2.15). The equation (2.11) is then no more meaningful since the quantity $\frac{\partial h_{\alpha}}{\partial t}$, appears on both side of the equality. Nevertheless the sum of the equations (2.11) for all the layers is still relevant and the boundary condition (2.15) leads to a global continuity equation for the total water height $H$

$$
\frac{\partial H}{\partial t}+\frac{\partial}{\partial x} \sum_{\alpha=1}^{N} h_{\alpha} u_{\alpha}=0,
$$

and each layer depth $h_{\alpha}$ is then deduced from the total water height by the relation

$$
h_{\alpha}=l_{\alpha} H,
$$

with $l_{\alpha}, \alpha=1, \ldots, N$ a given number satisfying

$$
l_{\alpha} \geq 0, \quad \sum_{\alpha=1}^{N} l_{\alpha}=1
$$

Thus the momentum equation (2.12) becomes

$$
\frac{\partial h_{\alpha} u_{\alpha}}{\partial t}+\frac{\partial}{\partial x}\left(h_{\alpha}\left\langle u^{2}\right\rangle_{\alpha}+\frac{1}{l_{\alpha}} \frac{g h_{\alpha}^{2}}{2}\right)=-g h_{\alpha} \frac{\partial z_{b}}{\partial x}+u_{\alpha+1 / 2} G_{\alpha+1 / 2}-u_{\alpha-1 / 2} G_{\alpha-1 / 2} .
$$

Using (2.18), (2.19), the expression of $G_{\alpha+1 / 2}$ given by (2.16) can also be written

$$
G_{\alpha+1 / 2}=\sum_{j=1}^{\alpha}\left(\frac{\partial h_{j} u_{j}}{\partial x}-l_{j} \sum_{i=1}^{N} \frac{\partial h_{i} u_{i}}{\partial x}\right) .
$$

Finally we have to define the quantities $h_{\alpha}\left\langle u^{2}\right\rangle_{\alpha}$ and $u_{\alpha+1 / 2}$ appearing in (2.12). As usual in the derivation of such systems, we have considered $h_{\alpha}\left\langle u^{2}\right\rangle_{\alpha} \approx h_{\alpha} u_{\alpha}^{2}$, this will be discussed in details in Section 3.5. The velocities $u_{\alpha+1 / 2}, \alpha=1, \ldots, N-1$ are obtained using an upwinding

$$
u_{\alpha+1 / 2}= \begin{cases}u_{\alpha} & \text { if } G_{\alpha+1 / 2} \geq 0 \\ u_{\alpha+1} & \text { if } G_{\alpha+1 / 2}<0 .\end{cases}
$$

To illustrate the formulation of the new model, we compare it with the system proposed in [1] in the simple case of a two-layer formulation. Neglecting the viscosity and friction, the formulation obtained by Audusse [1] 
corresponds to (2.11), (2.12) with (2.17), i.e.

$$
\begin{aligned}
& \frac{\partial h_{1}}{\partial t}+\frac{\partial h_{1} u_{1}}{\partial x}=0, \quad \frac{\partial h_{2}}{\partial t}+\frac{\partial h_{2} u_{2}}{\partial x}=0, \\
& \frac{\partial h_{1} u_{1}}{\partial t}+\frac{\partial h_{1} u_{1}^{2}}{\partial x}+g h_{1} \frac{\partial\left(h_{1}+h_{2}\right)}{\partial x}=-g h_{1} \frac{\partial z_{b}}{\partial x}, \\
& \frac{\partial h_{2} u_{2}}{\partial t}+\frac{\partial h_{2} u_{2}^{2}}{\partial x}+g h_{2} \frac{\partial\left(h_{1}+h_{2}\right)}{\partial x}=-g h_{2} \frac{\partial z_{b}}{\partial x},
\end{aligned}
$$

with $h_{1}+h_{2}=H$. The preceding formulation corresponds to a superposition of two single layer Saint-Venant systems (see also $[9,12,13]$ where a very similar model is considered in a bi-fluid framework).

With our approach (2.18), (2.21), the two-layer formulation reads

$$
\begin{aligned}
& \frac{\partial H}{\partial t}+\frac{\partial h_{1} u_{1}}{\partial x}+\frac{\partial h_{2} u_{2}}{\partial x}=0, \\
& \frac{\partial h_{1} u_{1}}{\partial t}+\frac{\partial h_{1} u_{1}^{2}}{\partial x}+\frac{g}{2} \frac{\partial H h_{1}}{\partial x}=-g h_{1} \frac{\partial z_{b}}{\partial x}+u_{3 / 2}\left(l \frac{\partial H}{\partial t}+l \frac{\partial H u_{1}}{\partial x}\right), \\
& \frac{\partial h_{2} u_{2}}{\partial t}+\frac{\partial h_{2} u_{2}^{2}}{\partial x}+\frac{g}{2} \frac{\partial H h_{2}}{\partial x}=-g h_{2} \frac{\partial z_{b}}{\partial x}-u_{3 / 2}\left(l \frac{\partial H}{\partial t}+l \frac{\partial H u_{1}}{\partial x}\right), \\
& \text { where } h_{1}=l H, \quad h_{2}=(1-l) H,
\end{aligned}
$$

with $l \in(0,1)$ prescribed. The velocity at the interface, denoted $u_{3 / 2}$, is calculated using upwinding, following the sign of the mass exchange between the layers. It is important to notice that, in the new formulation (2.27)(2.30), we obtain directly a left hand side term written in conservative form with the topography and the mass exchange as source terms whereas the pressure term of (2.24)-(2.26) has to be modified [1] to get a conservative form. Moreover we prove in Section 4 that the system (2.27)-(2.30) is hyperbolic, which is not the case for system (2.24)-(2.26).

The difference between (2.27)-(2.30) and (2.24)-(2.26) mainly comes from the physical definition of the layers. Audusse introduces a physical discretization where each layer has its own continuity equation. These $N$ continuity equations mean the layers are isolated each other, this situation corresponds to the case of $N$ non miscible fluids. In the formulation (2.27)-(2.30), the discretization corresponds to a semidiscretization in the vertical direction - of $P_{0}$ finite elements type - of the velocity $u$. In this case, the definition of the layers does not correspond to a physical partition of the flow but is related to the quality of the desired approximation over $u$. Thus we have only one continuity equation meaning the fluid can circulate from one layer to another.

\section{Derivation of the viscous Multilayer SHALlow WATER System}

In this section we will apply to the Navier-Stokes equations the multilayer approach presented in the preceding section.

\subsection{The Navier-Stokes equations}

Let us start with the incompressible Navier-Stokes system [19] restricted to two dimensions with gravity in which the $z$ axis represents the vertical direction. For simplicity, the viscosity will be kept constant and isotropic throughout the paper (we refer the reader to [15] for a more general framework). Therefore we have 
the following general formulation:

$$
\begin{aligned}
& \frac{\partial u}{\partial x}+\frac{\partial w}{\partial z}=0 \\
& \frac{\partial u}{\partial t}+u \frac{\partial u}{\partial x}+w \frac{\partial u}{\partial z}+\frac{\partial p}{\partial x}=\frac{\partial \Sigma_{x x}}{\partial x}+\frac{\partial \Sigma_{x z}}{\partial z} \\
& \frac{\partial w}{\partial t}+u \frac{\partial w}{\partial x}+w \frac{\partial w}{\partial z}+\frac{\partial p}{\partial z}=-g+\frac{\partial \Sigma_{z x}}{\partial x}+\frac{\partial \Sigma_{z z}}{\partial z}
\end{aligned}
$$

and we consider this system for

$$
t>t_{0}, \quad x \in \mathbb{R}, \quad z_{b}(x, t) \leq z \leq \eta(x, t) .
$$

We use the same notations as in the previous section. We now consider the bathimetry $z_{b}$ can vary with respect to abscissa $x$ and also with respect to time $t$. The chosen form of the viscosity tensor is symmetric

$$
\begin{array}{ll}
\Sigma_{x x}=2 \nu \frac{\partial u}{\partial x}, & \Sigma_{x z}=\nu\left(\frac{\partial u}{\partial z}+\frac{\partial w}{\partial x}\right), \\
\Sigma_{z z}=2 \nu \frac{\partial w}{\partial z}, & \Sigma_{z x}=\nu\left(\frac{\partial u}{\partial z}+\frac{\partial w}{\partial x}\right),
\end{array}
$$

with $\nu$ the viscosity coefficient.

\subsection{Boundary conditions}

The system (3.31)-(3.33) is complete with boundary conditions. The outward and upward unit normals to the free surface $\mathbf{n}_{s}$ and to the bottom $\mathbf{n}_{b}$ are given by

$$
\mathbf{n}_{s}=\frac{1}{\sqrt{1+\left(\frac{\partial \eta}{\partial x}\right)^{2}}}\left(\begin{array}{c}
-\frac{\partial \eta}{\partial x} \\
1
\end{array}\right), \quad \mathbf{n}_{b}=\frac{1}{\sqrt{1+\left(\frac{\partial z_{b}}{\partial x}\right)^{2}}}\left(\begin{array}{c}
-\frac{\partial z_{b}}{\partial x} \\
1
\end{array}\right) .
$$

Let $\Sigma_{T}$ be the total stress tensor with

$$
\Sigma_{T}=-p I_{d}+\left(\begin{array}{cc}
\Sigma_{x x} & \Sigma_{x z} \\
\Sigma_{z x} & \Sigma_{z z}
\end{array}\right)
$$

At the free surface we have the kinematic boundary condition (2.4). Considering the air viscosity is negligible, the continuity of stresses at the free boundary imposes

$$
\Sigma_{T} \mathbf{n}_{s}=-p^{a} \mathbf{n}_{s}
$$

where $p^{a}=p^{a}(x, t)$ is a given function corresponding to the atmospheric pressure. Relation (3.34) is equivalent to

$$
\mathbf{n}_{s} \cdot \Sigma_{T} \mathbf{n}_{s}=-p^{a}, \quad \text { and } \quad \mathbf{t}_{s} \cdot \Sigma_{T} \mathbf{n}_{s}=0
$$

$\mathbf{t}_{s}$ being orthogonal to $\mathbf{n}_{s}$.

Since we now consider the bottom can vary with respect to time $t$, the kinematic boundary condition reads

$$
\frac{\partial z_{b}}{\partial t}+u_{b} \frac{\partial z_{b}}{\partial x}-w_{b}=0,
$$


where $(x, t) \mapsto z_{b}(x, t)$ is a given function. Notice that equation (3.35) reduces to a classical no-penetration condition (2.5) when $z_{b}$ does not depend on time $t$. For the stresses at the bottom we consider a wall law under the form

$$
\Sigma_{T} \mathbf{n}_{b}-\left(\mathbf{n}_{b} . \Sigma_{T} \mathbf{n}_{b}\right) \mathbf{n}_{b}=\kappa\left(\mathbf{v}_{\mathbf{b}}, H\right) \mathbf{v}_{b},
$$

with $\mathbf{v}_{b}=\mathbf{u}_{b}-\left(0, \frac{\partial z_{b}}{\partial t}\right)^{T}$ the relative velocity between the water and the bottom. If $\kappa\left(\mathbf{v}_{\mathbf{b}}, H\right)$ is constant then we recover a Navier friction condition as in [18]. Introducing $k_{l}$ laminar and $k_{t}$ turbulent friction coefficients, we use the expression

$$
\kappa\left(\mathbf{v}_{\mathbf{b}}, H\right)=k_{l}+k_{t} H\left|\mathbf{v}_{\mathbf{b}}\right|
$$

corresponding to the boundary condition used in [20]. Another form of $\kappa\left(\mathbf{v}_{\mathbf{b}}, H\right)$ is used in [10] and for other wall laws, the reader can also refer to [21]. Due to thermomechanical considerations, in the sequel we suppose $\kappa\left(\mathbf{v}_{\mathbf{b}}, H\right) \geq 0$ and $\kappa\left(\mathbf{v}_{\mathbf{b}}, H\right)$ is often simply denoted by $\kappa$.

Let $\mathbf{t}_{b}$ satisfying $\mathbf{t}_{b} \cdot \mathbf{n}_{b}=0$ then when multiplied by $\mathbf{t}_{b}$ and $\mathbf{n}_{b}$, equation (3.36) leads to

$$
\mathbf{t}_{b} \cdot \Sigma_{T} \mathbf{n}_{b}=\kappa \mathbf{v}_{b} \cdot \mathbf{t}_{b}, \quad \text { and } \quad \mathbf{v}_{b} \cdot \mathbf{n}_{b}=0 .
$$

\subsection{The rescaled system}

The physical system is rescaled using the quantities:

- $h$ and $\lambda$, two characteristic dimensions along the $z$ and $x$ axis respectively;

- $a_{s}$ the typical wave amplitude, $a_{b}$ the typical bathimetry variation;

- $C=\sqrt{g h}$ the typical horizontal wave speed.

Classically for the derivation of the Saint-Venant system, we introduce the small parameter

$$
\varepsilon=\frac{h}{\lambda}
$$

When considering long waves propagation, another important parameter needs to be considered, namely

$$
\delta=\frac{a_{s}}{h}
$$

and we consider for the bathimetry $\frac{a_{b}}{h}=\mathcal{O}(\delta)$. Notice that $\varepsilon$ is related to a priori informations only associated to geometrical features whereas $a_{s}$ and accordingly $\delta$ deal with the state variables of the problem.

Depending on the application, $\delta$ can be considered or not as a small parameter. For finite amplitude wave theory and assuming $z_{b}(x, t)=z_{b}^{0}$, one considers $\varepsilon \ll 1, \delta=\mathcal{O}(1)$ whereas the Boussinesq waves theory requires

$$
\delta \ll 1, \quad \varepsilon \ll 1 \text { and } \quad U_{r}=\mathcal{O}(1)
$$

where $U_{r}$ is the Ursell number defined by $U_{r}=\frac{\delta}{\varepsilon^{2}}$, see [28]. All along this work, we consider $\varepsilon \ll 1$ whereas, even if the parameter $\delta$ is introduced in the rescaling, the assumption $\delta \ll 1$ is not considered except when explicitly mentioned.

As for the Saint-Venant system [18,20], we introduce some characteristic quantities: $T=\lambda / C$ for the time, $W=a_{s} / T=\varepsilon \delta C$ for the vertical velocity, $U=W / \varepsilon=\delta C$, for the horizontal velocity, $P=C^{2}$ for the pressure. This leads to the following dimensionless quantities

$$
\begin{aligned}
& \tilde{x}=\frac{x}{\lambda}, \quad \tilde{z}=\frac{z}{h}, \quad \tilde{\eta}=\frac{\eta}{a_{s}}, \quad \tilde{t}=\frac{t}{T}, \\
& \tilde{p}=\frac{p}{P}, \quad \tilde{u}=\frac{u}{U}, \quad \text { and } \quad \tilde{w}=\frac{w}{W} .
\end{aligned}
$$

Notice that the definition of the characteristic velocities implies $\delta=\frac{U}{C}$ so $\delta$ also corresponds to the Froude number. When $\delta=\mathcal{O}(1)$ we have $U \approx C$ and we recover the classical rescaling used for the Saint-Venant 
system. For the bathimetry $z_{b}$ we write $z_{b}(x, t)=Z_{b}(x)+b(t)$ and we introduce $\tilde{z}_{b}=Z_{b} / h$ and $\tilde{b}=b / a_{b}$. This leads to

$$
\frac{\partial z_{b}}{\partial t}=\varepsilon \delta C \frac{\partial \tilde{b}}{\partial \tilde{t}}=W \frac{\partial \tilde{b}}{\partial \tilde{t}}, \quad \text { and } \quad \frac{\partial z_{b}}{\partial x}=\varepsilon \frac{\partial \tilde{z}_{b}}{\partial \tilde{x}} .
$$

The different rescaling applied to the time and space derivatives of $z_{b}$ means that a classical shallow water assumption is made concerning the space variations of the bottom profile whereas we assume the time variations of $z_{b}$ lie in the framework of the Boussinesq assumption and are consistent with the rescaling applied to the velocity $w$.

We also introduce $\tilde{\nu}=\frac{\nu}{\lambda C}$ and we set $\tilde{\kappa}=\frac{\kappa}{C}$. Notice that the definitions for the dimensionless quantities are consistent with the one used for the Boussinesq system [23,29]. Notice also that the rescaling used by Nwogu [22] differs from the preceding one since Nwogu uses $\tilde{w}=\frac{\varepsilon^{2}}{W} w$.

As in $[18,20]$, we suppose we are in the following asymptotic regime

$$
\tilde{\nu}=\varepsilon \nu_{0}, \quad \text { and } \quad \tilde{\kappa}=\varepsilon \kappa_{0},
$$

with $\kappa_{0}=\kappa_{l, 0}+\varepsilon \kappa_{t, 0}\left(\tilde{\mathbf{v}}_{b}, \tilde{H}\right), \kappa_{l, 0}$ being constant.

This non-dimensionalization of the Navier-Stokes system (3.31)-(3.33) leads to

$$
\begin{aligned}
& \frac{\partial \tilde{u}}{\partial \tilde{x}}+\frac{\partial \tilde{w}}{\partial \tilde{z}}=0, \\
& \varepsilon \delta \frac{\partial \tilde{u}}{\partial \tilde{t}}+\varepsilon \delta^{2} \frac{\partial \tilde{u}^{2}}{\partial \tilde{x}}+\varepsilon \delta^{2} \frac{\partial \tilde{u} \tilde{w}}{\partial \tilde{z}}+\varepsilon \frac{\partial \tilde{p}}{\partial \tilde{x}}=\varepsilon^{2} \delta \frac{\partial}{\partial \tilde{x}}\left(2 \nu_{0} \frac{\partial \tilde{u}}{\partial \tilde{x}}\right)+\frac{\partial}{\partial \tilde{z}}\left(\delta \nu_{0} \frac{\partial \tilde{u}}{\partial \tilde{z}}+\varepsilon^{2} \delta \nu_{0} \frac{\partial \tilde{w}}{\partial \tilde{x}}\right), \\
& \varepsilon^{2} \delta\left(\frac{\partial \tilde{w}}{\partial \tilde{t}}+\delta \frac{\partial \tilde{u} \tilde{w}}{\partial \tilde{x}}+\delta \frac{\partial \tilde{w}^{2}}{\partial \tilde{z}}\right)+\frac{\partial \tilde{p}}{\partial \tilde{z}}=-1+\frac{\partial}{\partial \tilde{x}}\left(\varepsilon \delta \nu_{0} \frac{\partial \tilde{u}}{\partial \tilde{z}}+\nu_{0} \varepsilon^{3} \delta \frac{\partial \tilde{w}}{\partial \tilde{x}}\right)+\varepsilon \delta \frac{\partial}{\partial \tilde{z}}\left(2 \nu_{0} \frac{\partial \tilde{w}}{\partial \tilde{z}}\right),
\end{aligned}
$$

where we use the divergence free condition to write velocity equations (3.38) and (3.39) in a conservative form. The associated boundary conditions (2.4), (3.34)-(3.36) become

$$
\begin{aligned}
& \frac{\partial \tilde{\eta}}{\partial \tilde{t}}+\delta \tilde{u}_{s} \frac{\partial \tilde{\eta}}{\partial \tilde{x}}-\tilde{w}_{s}=0 \\
& \left.2 \varepsilon \delta \nu_{0} \frac{\partial \tilde{w}}{\partial \tilde{z}}\right|_{s}-\tilde{p}_{s}-\varepsilon \delta^{2} \nu_{0} \frac{\partial \tilde{\eta}}{\partial \tilde{x}}\left(\left.\frac{\partial \tilde{u}}{\partial \tilde{z}}\right|_{s}+\left.\varepsilon^{2} \frac{\partial \tilde{w}}{\partial \tilde{x}}\right|_{s}\right)=-\delta \tilde{p}^{a} \\
& \delta \nu_{0}\left(\left.\frac{\partial \tilde{u}}{\partial \tilde{z}}\right|_{s}+\left.\varepsilon^{2} \frac{\partial \tilde{w}}{\partial \tilde{x}}\right|_{s}\right)-\varepsilon \delta \frac{\partial \tilde{\eta}}{\partial \tilde{x}}\left(\left.2 \varepsilon \delta \nu_{0} \frac{\partial \tilde{u}}{\partial \tilde{x}}\right|_{s}-\tilde{p}_{s}\right)=\varepsilon \delta^{2} \frac{\partial \tilde{\eta}}{\partial \tilde{x}} \tilde{p}^{a}, \\
& \frac{\partial \tilde{b}}{\partial \tilde{t}}+\tilde{u}_{b} \frac{\partial \tilde{z}_{b}}{\partial \tilde{x}}-\tilde{w}_{b}=0 \\
& \delta \nu_{0}\left(\left.\varepsilon^{2} \frac{\partial \tilde{w}}{\partial \tilde{x}}\right|_{b}+\left.\frac{\partial \tilde{u}}{\partial \tilde{z}}\right|_{b}\right)-\varepsilon \frac{\partial \tilde{z} b}{\partial \tilde{x}}\left(\left.2 \varepsilon \delta \nu_{0} \frac{\partial \tilde{u}}{\partial \tilde{x}}\right|_{b}-p_{b}\right)+\varepsilon \frac{\partial \tilde{z}_{b}}{\partial \tilde{x}}\left(\left.2 \varepsilon \delta \nu_{0} \frac{\partial \tilde{w}}{\partial \tilde{z}}\right|_{b}-p_{b}\right. \\
& \left.\quad-\varepsilon \nu_{0} \frac{\partial \tilde{z}_{b}}{\partial \tilde{x}}\left(\left.\delta \frac{\partial \tilde{u}}{\partial \tilde{z}}\right|_{b}+\left.\varepsilon^{2} \delta \frac{\partial \tilde{w}}{\partial \tilde{x}}\right|_{b}\right)\right)=\varepsilon \delta \kappa_{0} \sqrt{1+\varepsilon^{2}\left(\frac{\partial \tilde{z}_{b}}{\partial \tilde{x}}\right)^{2}}\left(\tilde{u}_{b}+\varepsilon^{2} \frac{\partial \tilde{z}_{b}}{\partial \tilde{x}}\left(\tilde{w}_{b}-\frac{\partial \tilde{b}}{\partial \tilde{t}}\right)\right) .
\end{aligned}
$$

For the sake of clarity, in the sequel we drop the symbol $\sim$ and we denote $\frac{\partial b}{\partial t}=\frac{\partial z_{b}}{\partial t}$.

\subsection{The shallow water system}

The derivation of multilayer approximation is somehow technical. In order to better explain the analysis we recall the monolayer case following the asymptotic expansion in [18]. 
In the following the two sets of equations (3.37)-(3.39) and (3.40)-(3.44) are approximated to retain only the high order terms.

Due to the applied rescaling some terms of the viscosity tensor e.g.

$$
\varepsilon^{3} \delta \frac{\partial}{\partial x}\left(\nu_{0} \frac{\partial w}{\partial x}\right)
$$

are very small and could be neglected. But, as mentioned in [1], Remarks 1 and 2, the approximation of the viscous terms has to preserve the dissipation energy that is an essential property of the Navier-Stokes and averaged Navier-Stokes equations. Since we privilege this stability requirement and in order to keep a symmetric form of the viscosity tensor, we consider in the sequel a modified version of (3.37)-(3.39) under the form

$$
\begin{aligned}
& \frac{\partial u}{\partial x}+\frac{\partial w}{\partial z}=0 \\
& \varepsilon \delta \frac{\partial u}{\partial t}+\varepsilon \delta^{2} \frac{\partial u^{2}}{\partial x}+\varepsilon \delta^{2} \frac{\partial u w}{\partial z}+\varepsilon \frac{\partial p}{\partial x}=\varepsilon^{2} \delta \frac{\partial}{\partial x}\left(2 \nu_{0} \frac{\partial u}{\partial x}\right)+\frac{\partial}{\partial z}\left(\delta \nu_{0} \frac{\partial u}{\partial z}\right) \\
& \varepsilon^{2} \delta\left(\frac{\partial w}{\partial t}+\delta \frac{\partial u w}{\partial x}+\delta \frac{\partial w^{2}}{\partial z}\right)+\frac{\partial p}{\partial z}=-1+\frac{\partial}{\partial x}\left(\varepsilon \delta \nu_{0} \frac{\partial u}{\partial z}\right)+\frac{\partial}{\partial z}\left(2 \varepsilon \delta \nu_{0} \frac{\partial w}{\partial z}\right)
\end{aligned}
$$

corresponding to a viscosity tensor of the form

$$
\Sigma_{x x}=2 \nu \frac{\partial u}{\partial x}, \quad \Sigma_{x z}=\Sigma_{z x}=\nu \frac{\partial u}{\partial z}, \quad \Sigma_{z z}=2 \nu \frac{\partial w}{\partial z} .
$$

This means the terms in $\varepsilon^{2} \partial_{x} w$ have been neglected in (3.37)-(3.39) and in (3.40)-(3.44). For details about the adopted form of the viscosity tensor see ([11], Rem. 2) and ([1], Lem. 2.1).

In the same way, retaining only the high order terms, the boundary conditions (3.40)-(3.44) become

$$
\begin{aligned}
& \frac{\partial \eta}{\partial t}+\delta u_{s} \frac{\partial \eta}{\partial x}-w_{s}=0, \\
& \left.2 \varepsilon \delta \nu_{0} \frac{\partial w}{\partial z}\right|_{s}-p_{s}-\left.\varepsilon \delta^{2} \nu_{0} \frac{\partial \eta}{\partial x} \frac{\partial u}{\partial z}\right|_{s}=-\delta p^{a}, \\
& \left.\delta \nu_{0} \frac{\partial u}{\partial z}\right|_{s}-\varepsilon \delta \frac{\partial \eta}{\partial x}\left(\left.2 \varepsilon \delta \nu_{0} \frac{\partial u}{\partial x}\right|_{s}-p_{s}\right)=\varepsilon \delta^{2} \frac{\partial \eta}{\partial x} p^{a}, \\
& \frac{\partial z_{b}}{\partial t}+u_{b} \frac{\partial z_{b}}{\partial x}-w_{b}=0, \\
& \left.\delta \nu_{0} \frac{\partial u}{\partial z}\right|_{b}-\varepsilon \frac{\partial z_{b}}{\partial x}\left(\left.2 \varepsilon \delta \nu_{0} \frac{\partial u}{\partial x}\right|_{b}-p_{b}\right)+\varepsilon \frac{\partial z_{b}}{\partial x}\left(\left.2 \varepsilon \delta \nu_{0} \frac{\partial w}{\partial z}\right|_{b}-p_{b}-\left.\varepsilon \delta \nu_{0} \frac{\partial z_{b}}{\partial x} \frac{\partial u}{\partial z}\right|_{b}\right) \\
& \quad=\varepsilon \delta \kappa_{0}\left(1+\varepsilon^{2}\left(\frac{\partial z_{b}}{\partial x}\right)^{2}\right)^{3 / 2} u_{b} .
\end{aligned}
$$

Now we will exhibit the hydrostatic and non hydrostatic parts of the pressure. An integration of (3.47) from $z$ to $\delta \eta$ gives

$$
\begin{aligned}
& \varepsilon^{2} \delta \int_{z}^{\delta \eta}\left(\frac{\partial w}{\partial t}+\delta \frac{\partial(u w)}{\partial x}\right) \mathrm{d} z+\varepsilon^{2} \delta^{2}\left(w_{s}^{2}-w^{2}\right)+p_{s}-p \\
& \quad=-(\delta \eta-z)+\varepsilon \delta \int_{z}^{\delta \eta} \frac{\partial}{\partial x}\left(\nu_{0} \frac{\partial u}{\partial z}\right) \mathrm{d} z-2 \varepsilon \delta \nu_{0} \frac{\partial w}{\partial z}+\left.2 \varepsilon \delta \nu_{0} \frac{\partial w}{\partial z}\right|_{s}
\end{aligned}
$$


From the equations (3.41) and (3.42) it comes

$$
\left.\frac{\partial u}{\partial z}\right|_{s}=\mathcal{O}\left(\varepsilon^{2}\right)
$$

and the boundary condition (3.41) gives

$$
p_{s}=\delta p^{a}+\left.2 \varepsilon \delta \frac{\partial w}{\partial z}\right|_{s}+\mathcal{O}\left(\varepsilon^{3} \delta^{2}\right)
$$

The previous relation and the kinematic boundary condition (3.40) allow us to rewrite (3.53) under the form

$$
\begin{aligned}
& \varepsilon^{2} \delta\left(\frac{\partial}{\partial t} \int_{z}^{\delta \eta} w \mathrm{~d} z+\delta \frac{\partial}{\partial x} \int_{z}^{\delta \eta}(u w) \mathrm{d} z\right)-\varepsilon^{2} \delta^{2} w^{2}+\delta p^{a}-p \\
& \quad=-(\delta \eta-z)+\varepsilon \delta \int_{z}^{\delta \eta} \frac{\partial}{\partial x}\left(\nu_{0} \frac{\partial u}{\partial z}\right) \mathrm{d} z-2 \varepsilon \delta \nu_{0} \frac{\partial w}{\partial z}+\mathcal{O}\left(\varepsilon^{3} \delta\right) .
\end{aligned}
$$

Classically we have

$$
\frac{\partial u_{s}}{\partial x}=\left.\frac{\partial u}{\partial x}\right|_{s}+\left.\delta \frac{\partial \eta}{\partial x} \frac{\partial u}{\partial z}\right|_{s}=\left.\frac{\partial u}{\partial x}\right|_{s}+\mathcal{O}\left(\varepsilon^{2} \delta\right)
$$

and using relations (3.45), (3.56) and the Leibniz rule we have

$$
\varepsilon \delta \int_{z}^{\delta \eta} \frac{\partial}{\partial x}\left(\nu_{0} \frac{\partial u}{\partial z}\right) \mathrm{d} z-2 \varepsilon \delta \nu_{0} \frac{\partial w}{\partial z}=\varepsilon \delta \nu_{0} \frac{\partial u}{\partial x}+\left.\varepsilon \delta \nu_{0} \frac{\partial u}{\partial x}\right|_{s}+\mathcal{O}\left(\varepsilon^{3} \delta\right)
$$

This leads to the expression for the pressure $p$

$$
p=p_{h}+p_{n h}+\mathcal{O}\left(\varepsilon^{3} \delta\right),
$$

where the viscous and hydrostatic part $p_{h}$ is given by

$$
p_{h}=\delta p^{a}+(\delta \eta-z)-\varepsilon \delta \nu_{0} \frac{\partial u}{\partial x}-\left.\varepsilon \delta \nu_{0} \frac{\partial u}{\partial x}\right|_{s}
$$

and the non-hydrostatic part $p_{n h}$ is

$$
p_{n h}=\varepsilon^{2} \delta\left(\frac{\partial}{\partial t} \int_{z}^{\delta \eta} w \mathrm{~d} z+\delta \frac{\partial}{\partial x} \int_{z}^{\delta \eta}(u w) \mathrm{d} z\right)-\varepsilon^{2} \delta^{2} w^{2} .
$$

The derivation and analysis of a classical Saint-Venant type system taking into account the non-hydrostatic part of the pressure has already been carried out by the authors [11]. The derivation of the multilayer system in this general framework is in progress. It will be presented in a forthcoming paper.

In the sequel, we restrict to the situation $p_{n h}=0$. Due to this hydrostatic assumption, we have

$$
p=p_{h}+\mathcal{O}\left(\varepsilon^{2} \delta\right)
$$

and we retain for $p_{h}$ the expression

$$
p_{h}=\delta p^{a}+(\delta \eta-z)-2 \varepsilon \delta \nu_{0} \frac{\partial u}{\partial x} .
$$


Then using (3.42), (3.44) and (3.55) one obtains

$$
\left.\frac{\partial u}{\partial z}\right|_{s}=\mathcal{O}\left(\varepsilon^{2}\right),\left.\quad \frac{\partial u}{\partial z}\right|_{b}=\mathcal{O}(\varepsilon) .
$$

From (3.58), (3.59) we can write

$$
p-\delta p^{a}=\delta \eta-z+\mathcal{O}(\varepsilon \delta),
$$

leading to

$$
\frac{\partial p}{\partial x}=\mathcal{O}(\delta) .
$$

The preceding relation inserted in (3.46) leads to

$$
\nu_{0} \frac{\partial^{2} u}{\partial z^{2}}=\mathcal{O}(\varepsilon)
$$

and equations (3.60) and (3.62) mean that

$$
u(x, z, t)=u(x, 0, t)+\mathcal{O}(\varepsilon),
$$

i.e. we recognize the so-called "motion by slices" of the usual Saint-Venant system. If we introduce the averaged quantity

$$
\bar{u}=\frac{1}{\delta \eta-z_{b}} \int_{z_{b}}^{\delta \eta} u \mathrm{~d} z
$$

it is well known $[11,16,18,20]$ that the shallow water system (3.45), (3.46) with an hydrostatic pressure (3.58), (3.59) is approximated in $\mathcal{O}\left(\varepsilon^{2} \delta\right)$ by the following Saint-Venant system written with the variables with dimension

$$
\begin{aligned}
& \frac{\partial H}{\partial t}+\frac{\partial H \bar{u}}{\partial x}=0, \\
& \frac{\partial H \bar{u}}{\partial t}+\frac{\partial H \bar{u}^{2}}{\partial x}+\frac{g}{2} \frac{\partial H^{2}}{\partial x}=-H \frac{\partial p^{a}}{\partial x}-g H \frac{\partial z_{b}}{\partial x}+\frac{\partial}{\partial x}\left(4 \nu H \frac{\partial \bar{u}}{\partial x}\right)-\frac{\kappa(\overline{\mathbf{v}}, H)}{1+\frac{\kappa(\overline{\mathbf{v}}, H)}{3 \nu} H} \bar{u}
\end{aligned}
$$

with $H=\eta-z_{b}$.

\subsection{The viscous multilayer shallow water system}

We again consider the shallow water system (3.45), (3.46) with an hydrostatic pressure (3.58), (3.59). Here another approximation is introduced concerning the velocity $u$, it is no more assumed constant along the vertical but is discretized in the $z$ direction using piecewise constant functions, see Figure 2. As introduced in Section 2 the interval $\left[z_{b}, \delta \eta\right]$ is divided into $N$ layers of thickness $h_{\alpha}$ and we use the definitions (2.19), (2.20). We write

$$
u^{m c}\left(x, z,\left\{z_{\alpha}\right\}, t\right)=\sum_{\alpha=1}^{N} 1_{\left[z_{\alpha-1 / 2}, z_{\alpha+1 / 2}\right]}(z) u_{\alpha}(x, t)
$$

with the velocities $u_{\alpha}, \alpha \in[1, \ldots, N]$ defined by $(2.8)$.

Notice that from (2.7) we have $z_{1 / 2}=z_{b}=\mathcal{O}(1)$ and $z_{N+1 / 2}=\delta \eta=\mathcal{O}(\delta)$. The difference of magnitude between $z_{1 / 2}$ and $z_{N+1 / 2}$ makes the assumption $\delta \ll 1$ difficult to integrate in the definition of the $\left\{z_{\alpha+1 / 2}\right\}$.

Now we try to quantify the error between $u$ and its piecewise approximation $u^{m c}$. First we notice that in absence of friction at the bottom and due to the shallow water assumption, the relations (3.60) become

$$
\left.\frac{\partial u}{\partial z}\right|_{s}=\left.\frac{\partial u}{\partial z}\right|_{b}=\mathcal{O}\left(\varepsilon^{2}\right)
$$


This means we can consider that except for the bottom layer, each layer inherits the approximation (3.67) i.e.

$$
\frac{\partial u}{\partial z}=\mathcal{O}\left(\varepsilon^{2}\right) \quad \text { for } z \geq z_{3 / 2}
$$

and therefore for all $\alpha>1$

$$
u(x, z, t)-u_{\alpha}(x, t)=\mathcal{O}\left(\varepsilon^{2}\right)
$$

or equivalently

$$
u(x, z, t)-u^{m c}\left(x, z,\left\{z_{\alpha}\right\}, t\right)=\mathcal{O}\left(\varepsilon^{2}\right), \quad \text { for } z \geq z_{3 / 2} .
$$

In the bottom layer we only have

$$
u(x, z, t)-u_{1}(x, t)=\mathcal{O}(\varepsilon),
$$

but as in [11,18], it can be proved that we have an approximation of the velocity through a parabolic correction

$$
u=\left(1+\frac{\varepsilon \kappa_{0}}{\nu_{0}}\left(z-z_{b}-\frac{\left(z-z_{b}\right)^{2}}{2 H}-\frac{H}{3}\right)\right) u_{1}+\mathcal{O}\left(\varepsilon^{2}\right),
$$

for $z \in\left[z_{1 / 2}, z_{3 / 2}\right]$. Using the discretization (2.7), (2.8) and (3.66) we claim

Proposition 3.1. The multilayer formulation of the Saint-Venant system defined by

$$
\begin{aligned}
& \frac{\partial H}{\partial t}+\sum_{\alpha=1}^{N} \frac{\partial h_{\alpha} u_{\alpha}}{\partial x}=0 \\
& \frac{\partial h_{1} u_{1}}{\partial t}+\frac{\partial h_{1} u_{1}^{2}}{\partial x}+\frac{g}{2 l_{1}} \frac{\partial h_{1}^{2}}{\partial x}=-h_{1} \frac{\partial p^{a}}{\partial x}-g h_{1} \frac{\partial z_{b}}{\partial x}+u_{3 / 2} G_{3 / 2}+\frac{\partial}{\partial x}\left(4 \nu h_{1} \frac{\partial u_{1}}{\partial x}\right) \\
& -4 \nu \frac{\partial z_{3 / 2}}{\partial x} \frac{\partial u_{3 / 2}}{\partial x}+2 \nu \frac{u_{2}-u_{1}}{h_{2}+h_{1}}-\kappa(\overline{\mathbf{v}}, H) u_{1}, \\
& \frac{\partial h_{\alpha} u_{\alpha}}{\partial t}+\frac{\partial h_{\alpha} u_{\alpha}^{2}}{\partial x}+\frac{g}{2 l_{\alpha}} \frac{\partial h_{\alpha}^{2}}{\partial x}=-h_{\alpha} \frac{\partial p^{a}}{\partial x}-g h_{\alpha} \frac{\partial z_{b}}{\partial x}+u_{\alpha+1 / 2} G_{\alpha+1 / 2}-u_{\alpha-1 / 2} G_{\alpha-1 / 2} \\
& +\frac{\partial}{\partial x}\left(4 \nu h_{\alpha} \frac{\partial u_{\alpha}}{\partial x}\right)-4 \nu\left[\frac{\partial z_{j}}{\partial x} \frac{\partial u_{j}}{\partial x}\right]_{j=\alpha-1 / 2}^{j=\alpha+1 / 2}+2 \nu \frac{u_{\alpha+1}-u_{\alpha}}{h_{\alpha+1}+h_{\alpha}}-2 \nu \frac{u_{\alpha}-u_{\alpha-1}}{h_{\alpha}+h_{\alpha-1}}, \\
& \text { for } \alpha \in\{2, \ldots, N-1\} \\
& \frac{\partial h_{N} u_{N}}{\partial t}+\frac{\partial h_{N} u_{N}^{2}}{\partial x}+\frac{g}{2 l_{N}} \frac{\partial h_{N}^{2}}{\partial x}=-h_{N} \frac{\partial p^{a}}{\partial x}-g h_{N} \frac{\partial z_{b}}{\partial x}-u_{N-1 / 2} G_{N-1 / 2} \\
& +\frac{\partial}{\partial x}\left(4 \nu h_{N} \frac{\partial u_{N}}{\partial x}\right)+4 \nu \frac{\partial z_{N-1 / 2}}{\partial x} \frac{\partial u_{N-1 / 2}}{\partial x}-2 \nu \frac{u_{N}-u_{N-1}}{h_{N}+h_{N-1}},
\end{aligned}
$$

with $h_{\alpha}=l_{\alpha} H(x, t)$ and $G_{\alpha+1 / 2}$ given by (2.16), results from a formal asymptotic approximation in $\mathcal{O}\left(\varepsilon^{2} \delta\right)$ coupled with a vertical discretization of the Navier-Stokes equations (3.37)-(3.39) with hydrostatic pressure.

Proof. The integration of the divergence equation (3.45) on each layer has been already performed in the proof of Proposition 2.1. We recall that the deduced layer mass equations (2.11) are not meaningful if no hypothesis is made concerning the mass exchange term $G_{\alpha+1 / 2}$ defined by (2.16). We thus consider a global mass equation (3.70) by adding them up. We can also directly integrate the divergence equation from bottom to free surface in order to obtain equation (3.70). 
We now consider the horizontal velocity equation (3.46) integrated over the interval $\left[z_{\alpha-1 / 2}, z_{\alpha+1 / 2}\right]$. Using for each layer an approximation similar to (3.68), (3.69), we prove that

$$
\frac{1}{h_{\alpha}} \int_{z_{\alpha-1 / 2}}^{z_{\alpha}+1 / 2} u^{2}(x, z, t) \mathrm{d} z=u_{\alpha}^{2}+\mathcal{O}\left(\varepsilon^{2}\right)
$$

In the context of the hydrostatic approximation, we assume that the pressure satisfies (3.58), (3.59). The treatment of the inviscid part of the pressure has already been presented in the proof of Proposition 2.1 where we have written for the gravitational part of the pressure

$$
\int_{z_{\alpha-1 / 2}}^{z_{\alpha}+1 / 2} \frac{\partial}{\partial x}(\delta \eta-z) \mathrm{d} z=\frac{1}{2 l_{\alpha}} \frac{\partial}{\partial x} h_{\alpha}^{2}+h_{\alpha} \frac{\partial z_{b}}{\partial x}
$$

Notice that it is also possible to write

$$
\int_{z_{\alpha-1 / 2}}^{z_{\alpha}+1 / 2} \frac{\partial}{\partial x}(\delta \eta-z) \mathrm{d} z=\frac{1}{2} \frac{\partial}{\partial x}\left[h_{\alpha}\left(2 \sum_{j=\alpha+1}^{N} h_{j}+h_{\alpha}\right)\right]-\frac{\partial z_{\alpha+1 / 2}}{\partial x} \sum_{j=\alpha+1}^{N} h_{j}+\frac{\partial z_{\alpha-1 / 2}}{\partial x} \sum_{j=\alpha}^{N} h_{j} .
$$

The expressions (3.74) and (3.75) lead to the same property for the complete model even if the hyperbolic part is modified. The second formulation seems more adapted to the physical description "by layers" of the system but leads to complementary source terms whose discretization is subtle. We will use and analyse (3.75) in a forthcoming paper. In the following we use (3.74).

The integration of the viscous part of the pressure leads to

$$
\int_{z_{\alpha-1 / 2}}^{z_{\alpha}+1 / 2} \frac{\partial}{\partial x}\left(2 \nu \frac{\partial u}{\partial x}\right)=\frac{\partial}{\partial x}\left(2 \nu h_{\alpha} \frac{\partial u_{\alpha}}{\partial x}\right)+2 \nu\left[\frac{\partial z_{j}}{\partial x} \frac{\partial u_{j}}{\partial x}\right]_{j=\alpha-1 / 2}^{j=\alpha+1 / 2}+\mathcal{O}\left(\varepsilon^{2} \delta\right)
$$

It remains to consider the viscous terms on the right hand side of (3.46). The first one is similar to the viscous part of the pressure term. For the second one, using finite differences along the vertical, we write

$$
\begin{aligned}
\int_{z_{\alpha-1 / 2}}^{z_{\alpha+1 / 2}} \frac{\partial}{\partial z}\left(\nu_{0} \frac{\partial u}{\partial z}\right) \mathrm{d} z & =\left.\nu_{0} \frac{\partial u}{\partial z}\right|_{z_{\alpha+1 / 2}}-\left.\nu_{0} \frac{\partial u}{\partial z}\right|_{z_{\alpha-1 / 2}} \\
& \approx 2 \nu \frac{u_{\alpha+1}-u_{\alpha}}{h_{\alpha+1}+h_{\alpha}}-2 \nu \frac{u_{\alpha}-u_{\alpha-1}}{h_{\alpha}+h_{\alpha-1}}
\end{aligned}
$$

and relation (3.72) follows. Notice that equations (3.71) and (3.73) are concerned with the evolution of the discharge in the lowest and uppest layers, respectively. The difference between equations (3.71) and (3.73) and the general equation (3.72) comes from the particular form of the viscous effect at the bottom and at the free surface.

Finally we drop the $\mathcal{O}\left(\varepsilon^{2} \delta\right)$ terms and recovering the variables with dimension, we obtain the system (3.70)$(3.73)$.

\section{Properties of the Multilayer SyStem}

In this paragraph we examine some properties of the model depicted in Proposition 3.1. We study its hyperbolicity and we exhibit an energy inequality and a kinetic interpretation of the system. 


\subsection{Hyperbolicity}

\subsubsection{Two layers model}

For the simplicity of the discussion we restrict in this subsection to the two-layer version of the multilayer model.

Let us first say some words about the multilayer system (2.24)-(2.26) introduced by Audusse [1]. This nonmiscible multilayer system was proved to be non-hyperbolic. In the general case the system exhibits complex eigenvalues. In the very simple case $u_{1}=u_{2}=u$ the eigenvalues of the hyperbolic part was shown to be equal to the classical barotropic eigenvalues of the monolayer shallow water system $u+\sqrt{g H}, u-\sqrt{g H}$ plus a baroclinic eigenvalue $u$ that is concerned with the interface waves. Nevertheless the system is not hyperbolic since $u$ is a double eigenvalue associated to a one-dimensional eigenspace. This lack of hyperbolicity may lead to the development of instabilities at the interface $[1,12]$. In [1] a technical trick is proposed to cure the problem. Here we can prove the well-posedness of the system.

Proposition 4.1. The two-layer version of the multilayer Saint-Venant system (3.70)-(3.73) is strictly hyperbolic when the total water height is strictly positive.

Proof. The two-layer version of the multilayer system depicted in Proposition 3.1 stands - we denote $u=u_{3 / 2}$ with $u=u_{1}$ or $u=u_{2}$ (see 2.23$)-$

$$
\begin{aligned}
& \frac{\partial H}{\partial t}+l \frac{\partial H u_{1}}{\partial x}+(1-l) \frac{\partial H u_{2}}{\partial x}=0 \\
& \frac{\partial H u_{1}}{\partial t}+\frac{\partial H u_{1}^{2}}{\partial x}+\frac{g}{2} \frac{\partial H^{2}}{\partial x}=-g H \frac{\partial z_{b}}{\partial x}+u\left(\frac{\partial H}{\partial t}+\frac{\partial H u_{1}}{\partial x}\right)-H \frac{\partial p^{a}}{\partial x}+\frac{2 \nu}{l H}\left(u_{2}-u_{1}\right)-\tilde{\kappa}(\overline{\mathbf{v}}, H) u_{1}, \\
& \frac{\partial H u_{2}}{\partial t}+\frac{\partial H u_{2}^{2}}{\partial x}+\frac{g}{2} \frac{\partial H^{2}}{\partial x}=-g H \frac{\partial z_{b}}{\partial x}+u\left(\frac{\partial H}{\partial t}+\frac{\partial H u_{2}}{\partial x}\right)-\frac{2 \nu}{(1-l) H}\left(u_{2}-u_{1}\right)-H \frac{\partial p^{a}}{\partial x}
\end{aligned}
$$

The previous formulation can be written under the quasi-linear form

$$
M(X) \frac{\partial X}{\partial t}+A(X) \frac{\partial X}{\partial x}=S(X)
$$

with

$$
\begin{aligned}
& X=\left(\begin{array}{l}
H \\
q_{1} \\
q_{2}
\end{array}\right), \quad M(X)=\left(\begin{array}{ccc}
1 & 0 & 0 \\
-u & 1 & 0 \\
-u & 0 & 1
\end{array}\right), \quad A(X)=\left(\begin{array}{ccc}
0 & l & (1-l) \\
g H-u_{1}^{2} & 2 u_{1}-u & 0 \\
g H-u_{2}^{2} & 0 & 2 u_{2}-u
\end{array}\right), \\
& S(X)=\left(\begin{array}{c}
-g H \frac{\partial z_{b}}{\partial x}+\frac{2 \nu}{l H}\left(u_{2}-u_{1}\right)-\tilde{\kappa}(\overline{\mathbf{v}}, H) u_{1}-H \frac{\partial p^{a}}{\partial x} \\
-g H \frac{\partial z_{b}}{\partial x}-\frac{2 \nu}{(1-l) H}\left(u_{2}-u_{1}\right)-H \frac{\partial p^{a}}{\partial x}
\end{array}\right)
\end{aligned}
$$

and $q_{i}=H u_{i}, i=\{1,2\}$.

The three eigenvalues of $M^{-1}(X) A(X)$ are the roots of $D(x)=\operatorname{det}(A-x M)=0$ with

$$
D(x)=-x \Pi_{i=1}^{2}\left(2 u_{i}-u-x\right)-l\left(2 u_{2}-u-x\right)\left(g H-u_{1}^{2}+u x\right)-(1-l)\left(2 u_{1}-u-x\right)\left(g H-u_{2}^{2}+u x\right) .
$$

Let us fix $H, u_{1}$ and $u_{2}$ in $\mathbb{R}$ and $l \in(0,1)$. Let us suppose $u_{1}<u_{2}$ with $u_{2}=u_{1}+\gamma^{2}$. We recall that the value of the interface velocity $u$ is taken equal to $u_{1}$ or $u_{2}$ following the direction of the exchange of mass between the two layers. 
Let us first suppose that $u=u_{1}$. Then we obviously have

$$
D\left(u_{1}\right)=-2 g H l \gamma^{2}<0, \quad D(-\infty)=+\infty, \quad D(+\infty)=-\infty,
$$

and some computations lead to

$$
D\left(\max \left(u_{2}=u_{1}+\gamma^{2}, u_{1}+2 l \gamma^{2}\right)\right)>0
$$

since $D\left(u_{2}\right)=(1-2 l) g H \gamma^{2}+l \gamma^{6}>0$ if $l \leq 1 / 2$ and $D\left(u_{1}+2 l \gamma^{2}\right)=2 l(1-l)(4 l-1) \gamma^{6}>0$ if $l>1 / 2$. It follows that $D(x)$ has three real and simple eigenvalues.

Let us now suppose that $u=u_{2}$. Then we have

$$
D\left(u_{2}\right)=2 g H(1-l) \gamma^{2}>0, \quad D(-\infty)=+\infty, \quad D(+\infty)=-\infty,
$$

and some computations lead to

$$
D\left(\min \left(u_{1}=u_{2}-\gamma^{2}, u_{2}-2(1-l) \gamma^{2}\right)\right)<0,
$$

since $D\left(u_{1}\right)=(1-2 l) g H \gamma^{2}-(1-l) \gamma^{6}<0$ if $l \geq 1 / 2$ and $D\left(u_{2}-2(1-l) \gamma^{2}\right)=2 l(1-l)(4 l-3) \gamma^{6}<0$ if $l<1 / 2$. Here also $D(x)$ has three real and simple eigenvalues.

The case $u_{2}<u_{1}$ is similar and we can conclude that the two-layer version of the multilayer system depicted in Proposition 3.1 is strictly hyperbolic. Notice that when $u_{1}=u_{2}=u$, we find the same baroclinic and barotropic eigenvalues $u, u+\sqrt{g H}, u-\sqrt{g H}$ as for the nonmiscible multilayer system [1], but they are all simple eigenvalues in this case since we consider a system with only three equations.

\subsubsection{General case}

In the case of $N$ layers the matrices $A(X)$ and $M(X)$ can be written

$$
\mathcal{A}_{N+1}=\left(\begin{array}{ccccc}
0 & l_{1} & \ldots & \ldots & l_{N} \\
g H-u_{1}^{2} & 2 u_{1} & \tilde{v}_{1,2} & \ldots & \tilde{v}_{1, N} \\
\vdots & \bar{v}_{2,1} & \ddots & \ddots & \vdots \\
\vdots & \vdots & \ddots & \ddots & \tilde{v}_{N-1, N} \\
g H-u_{N}^{2} & \bar{v}_{N, 1} & \ldots & \bar{v}_{N, N-1} & 2 u_{N}
\end{array}\right),
$$

with $\bar{v}_{i j}=u_{i-1 / 2} * l_{j} / l_{i}$ and $\tilde{v}_{i j}=u_{i+1 / 2} * l_{j} / l_{i}$,

$$
\mathcal{M}_{N+1}=\left(\begin{array}{ccccc}
1 & 0 & \ldots & \ldots & 0 \\
v_{1} & 1 & \ddots & & \vdots \\
\vdots & 0 & \ddots & \ddots & \vdots \\
\vdots & \vdots & \ddots & 1 & 0 \\
v_{N} & 0 & \ldots & 0 & 1
\end{array}\right)
$$

with $v_{i}=u_{i-1 / 2} * \sum_{j=1}^{i-1} l_{j} / l_{i}+u_{i+1 / 2} * \sum_{j=i+1}^{N} l_{j} / l_{i}$.

We have performed various numerical evaluations of the eigenelements of the matrix $\mathcal{M}_{N+1}^{-1} \mathcal{A}_{N+1}$ with numerous choices of the parameters $H, u_{\alpha}, u_{\alpha+1 / 2}$ and $l_{\alpha}$. When the parameters correspond to physical values, these tests have always shown that the matrix is diagonalizable on $\mathbb{R}$. But when considering shear flows with very large velocities e.g. $\left|u_{i_{0}}\right| \gg 1,\left|u_{j_{0}}\right| \gg 1$ and $u_{i_{0}} u_{j_{0}}<0$ for some $i_{0} \neq j_{0}$, complex eigenvalues can appear. Such a behavior is natural since neglecting the viscous and friction effects and as demonstrated in Proposition 3.1, our multilayer system approximates the hydrostatic free surface Euler system that is not an hyperbolic 
conservation law. Notice also that the kinetic scheme instead of using discrete eigenvalues of the Jacobian matrix, uses a continuum of eigenvalues and is able to deal with situations where some of the discrete eigenvalues are complex.

In the simple case where all the layers have the same velocity $u$, the barotropic eigenvalues $u+\sqrt{g H}$ and $u-\sqrt{g H}$ are simple and the baroclinic eigenvalue $u$ has a multiplicity of $N-1$ but the matrix remains diagonalizable on $\mathbb{R}$ and the problem is still well-posed.

\subsection{Energy equality}

The classical Saint-Venant system (3.64)-(3.65) admits an energy equality [1,11] under the form

$$
\frac{\partial E_{s v}}{\partial t}+\frac{\partial}{\partial x}\left(\bar{u}\left(E_{s v}+g \frac{H^{2}}{2}\right)-4 \nu H \bar{u} \frac{\partial \bar{u}}{\partial x}\right)=H \frac{\partial p^{a}}{\partial t}-4 \nu H\left(\frac{\partial \bar{u}}{\partial x}\right)^{2}-\frac{\kappa(\overline{\mathbf{v}}, H)}{1+\frac{\kappa(\overline{\mathbf{v}}, H) H}{3 \nu}} \bar{u}^{2}+g H \frac{\partial z_{b}}{\partial t}
$$

with $E_{s v}=\frac{H \bar{u}^{2}}{2}+\frac{g H\left(\eta+z_{b}\right)}{2}+H p^{a}$. Here we have the following result.

Proposition 4.2. For the multilayer Saint-Venant system (3.70)-(3.73), smooth solutions satisfy the energy equality

$$
\begin{aligned}
& \frac{\partial}{\partial t}\left(\sum_{\alpha=1}^{N} E_{s v, \alpha}^{m c}\right)+\frac{\partial}{\partial x}\left(\sum_{\alpha=1}^{N} u_{\alpha}\left(E_{s v, \alpha}^{m c}+\frac{g}{2} h_{\alpha} H-4 \nu h_{\alpha} \frac{\partial u_{\alpha}}{\partial x}\right)\right) \\
& \quad=-\kappa(\overline{\mathbf{v}}, H) u_{1}^{2}-\frac{\nu}{h_{\alpha}} \sum_{\alpha=1}^{N-1}\left(u_{\alpha+1 / 2}-u_{\alpha-1 / 2}\right)^{2}-4 \nu \sum_{\alpha=1}^{N} h_{\alpha}\left(\frac{\partial u_{\alpha}}{\partial x}\right)^{2}+H \frac{\partial p^{a}}{\partial t}+g H \frac{\partial z_{b}}{\partial t},
\end{aligned}
$$

with $E_{s v, \alpha}^{m c}=\frac{h_{\alpha} u_{\alpha}^{2}}{2}+\frac{g h_{\alpha}\left(\eta+z_{b}\right)}{2}+h_{\alpha} p^{a}$.

Proof. The proof relies on classical computations. Starting from (3.46) with $u=u^{m c}, p=p_{h}$ multiplying it with $u^{m c}$ and integrating over $\left[z_{\alpha-1 / 2}, z_{\alpha+1 / 2}\right]$ with $1<\alpha<N$ we obtain

$$
\begin{aligned}
& \frac{\partial}{\partial t} E_{s v, \alpha}^{m c}+\frac{\partial}{\partial x}\left(u_{\alpha}\left(E_{s v, \alpha}^{m c}+\frac{g}{2} h_{\alpha} H-4 \nu h \frac{\partial u_{\alpha}}{\partial x}\right)\right)=-\left(\frac{u_{\alpha-1 / 2}^{2}}{2}+p^{a}\right) G_{\alpha-1 / 2}+\left(\frac{u_{\alpha+1 / 2}^{2}}{2}+p^{a}\right) G_{\alpha+1 / 2} \\
& \quad-\left.\nu_{0} u_{\alpha-1 / 2} \frac{\partial u^{m c}}{\partial z}\right|_{z_{\alpha-1 / 2}}+\left.\nu_{0} u_{\alpha+1 / 2} \frac{\partial u^{m c}}{\partial z}\right|_{z_{\alpha+1 / 2}}+\left.\nu_{0} \frac{\partial z_{\alpha-1 / 2}}{\partial x} u_{\alpha-1 / 2} \frac{\partial u^{m c}}{\partial x}\right|_{z_{\alpha-1 / 2}} \\
& \quad-\left.\nu_{0} \frac{\partial z_{\alpha+1 / 2}}{\partial x} u_{\alpha+1 / 2} \frac{\partial u^{m c}}{\partial x}\right|_{z_{\alpha+1 / 2}}-\frac{\nu}{h_{\alpha}}\left(u_{\alpha+1 / 2}-u_{\alpha-1 / 2}\right)^{2}-4 \nu h_{\alpha}\left(\frac{\partial u_{\alpha}}{\partial x}\right)^{2}+h_{\alpha} \frac{\partial p^{a}}{\partial t}+g h_{\alpha} \frac{\partial z_{b}}{\partial t}, \quad(4.78)
\end{aligned}
$$

where we have considered for $z \in\left[z_{\alpha-1 / 2}, z_{\alpha+1 / 2}\right]$

$$
\frac{\partial u}{\partial z}=\frac{1}{h_{\alpha}}\left(u_{\alpha+1 / 2}-u_{\alpha-1 / 2}\right) .
$$

An analogous calculation is valid for $\alpha=1$ and $\alpha=N$. A sum from $\alpha=1$ to $\alpha=N$ of the equalities (4.78) with the boundary conditions (3.48)-(3.52) completes the proof.

\subsection{Kinetic interpretation}

For the simulation of a multilayer system several strategies are possible. Castro et al. [13] consider the full system and build a specific solver for the two-layer case. Following the discrete multilayer scheme proposed by Audusse [1] we prefer to exhibit a kinetic formulation of the system obtained in Proposition 3.1. Indeed kinetic 
schemes might be one of the best compromise between accuracy, stability and efficiency for the resolution of Saint-Venant type equations, see $[3,24]$. We refer to the next section for the presentation of the numerical scheme. Here we focus on the kinetic interpretation of the system.

The kinetic approach consists in using a description of the microscopic behavior of the system. In this method, fictitious particles are introduced and the equations are considered at the microscopic scale, where no discontinuities occur. The process to obtain the kinetic interpretation of the multilayer model is similar to the one used in [3] for the monolayer shallow water system. For a given layer $\alpha$, a distribution function $M_{\alpha}(x, t, \xi)$ of fictitious particles with microscopic velocity $\xi$ is introduced to obtain a linear microscopic kinetic equation equivalent to the macroscopic model presented in Proposition 3.1.

Let us introduce a real function $\chi$ defined on $\mathbb{R}$, compactly supported and which have the following properties

$$
\left\{\begin{array}{l}
\chi(-w)=\chi(w) \geq 0 \\
\int_{\mathbb{R}} \chi(w) \mathrm{d} w=\int_{\mathbb{R}} w^{2} \chi(w) \mathrm{d} w=1 .
\end{array}\right.
$$

Now let us construct a density of particles $M_{\alpha}(x, t, \xi)$ defined by a Gibbs equilibrium: the microscopic density of particles present at time $t$ in the layer $\alpha$, in the vicinity $\Delta x$ of the abscissa $x$ and with velocity $\xi$ given by

$$
M_{\alpha}(x, t, \xi)=l_{\alpha} \frac{H(x, t)}{c} \chi\left(\frac{\xi-u_{\alpha}(x, t)}{c}\right), \quad \alpha=1, \ldots, N,
$$

with

$$
c^{2}=\frac{g H}{2}
$$

Likewise, we define $N_{\alpha+1 / 2}(x, t, \xi)$ by

$$
N_{\alpha+1 / 2}(x, t, \xi)=G_{\alpha+1 / 2}(x, t) \delta\left(\xi-u_{\alpha+1 / 2}(x, t)\right), \quad \alpha=0, \ldots, N,
$$

where $\delta$ denotes the Dirac distribution. The quantities $G_{\alpha+1 / 2}, 0 \leq \alpha \leq N$ represent the mass exchanges between layers $\alpha$ and $\alpha+1$, they are defined in (2.16) and satisfy the conditions (2.15), so $N_{1 / 2}$ and $N_{N+1 / 2}$ also satisfy

$$
N_{1 / 2}(x, t, \xi)=N_{N+1 / 2}(x, t, \xi)=0 .
$$

We also introduce the densities $\widetilde{M}_{\alpha}(x, t, \xi)$ that will be used for the energy equations, they are defined by

$$
\widetilde{M}_{\alpha}(x, t, \xi)=\frac{g H(x, t) h_{\alpha}(x, t)}{4 c} \chi\left(\frac{\xi-u_{\alpha}(x, t)}{c}\right) .
$$

Notice that the introduction of this second family of densities is not needed when we consider the two dimensional shallow water system. Here they take into account some kind of transversal effect at the kinetic level that is implicitly included into the macroscopic one dimensional shallow water system. We refer the reader to $[3,25]$ for more details.

With the previous definitions, dropping the viscous, and friction terms, we write a kinetic representation of the multilayer Saint-Venant system described in Proposition 3.1 and we have the following proposition:

Proposition 4.3. The functions $\left(H, u^{m c}\right)$ are strong solutions of the multilayer Saint-Venant system (3.70)-(3.73) if and only if the set of equilibria $\left\{M_{\alpha}(x, t, \xi)\right\}_{\alpha=1}^{N}$ is solution of the kinetic equations

$$
\begin{aligned}
\frac{\partial M_{\alpha}}{\partial t}+\xi \frac{\partial M_{\alpha}}{\partial x}-\frac{\partial}{\partial x}\left(p^{a}+g z_{b}\right) \frac{\partial M_{\alpha}}{\partial \xi}-N_{\alpha+1 / 2}(x, t, \xi)+N_{\alpha-1 / 2}(x, t, \xi)= & Q_{\alpha}(x, t, \xi), \\
& \alpha=1, \ldots, N,
\end{aligned}
$$


with $\left\{N_{\alpha+1 / 2}(x, t, \xi)\right\}_{\alpha=0}^{N}$ satisfying (4.81), (4.82). The set of equations (4.83) can also be written under the form

$$
N_{\alpha+1 / 2}(x, t, \xi)=\sum_{i=1}^{\alpha}\left(\frac{\partial M_{i}}{\partial t}+\xi \frac{\partial M_{i}}{\partial x}-\frac{\partial}{\partial x}\left(p^{a}+z_{b}\right) \frac{\partial M_{i}}{\partial \xi}-Q_{i}\right), \quad \alpha=1, \ldots, N .
$$

The quantities $Q_{\alpha}(x, t, \xi)$ are "collision terms" equals to zero at the macroscopic level i.e. which satisfy for a.e. values of $(x, t)$

$$
\int_{\mathbb{R}} Q_{\alpha} \mathrm{d} \xi=0, \quad \int_{\mathbb{R}} \xi Q_{\alpha} \mathrm{d} \xi=0 .
$$

The solution of (4.83), (4.84) is an entropy solution if additionally

$$
\frac{\partial \widetilde{M}_{\alpha}}{\partial t}+\xi \frac{\partial \widetilde{M}_{\alpha}}{\partial x}=\widetilde{Q}_{\alpha}(x, t, \xi), \quad \alpha=1, \ldots, N
$$

with

$$
\int_{\mathbb{R}}\left(\frac{\xi^{2}}{2} Q_{\alpha}+\widetilde{Q}_{\alpha}\right) \mathrm{d} \xi \leq 0
$$

Proof. As previously we denote $X=\left(H, q_{1}, \ldots, q_{N}\right)^{T}$ the vector of unknowns with $q_{\alpha}=l_{\alpha} H u_{\alpha}$. We introduce $M=\left(M_{1}, \ldots, M_{N}\right)^{T}$ and an $(N+1) \times N$ matrix $\mathcal{K}(\xi)$ defined by $\mathcal{K}_{1, j}=1, \mathcal{K}_{i+1, j}=\delta_{i, j} \xi$ with $\delta_{i, j}$ the Kronecker symbol.

Using the definition (4.80) and the properties of the function $\chi$, we have

$$
l_{\alpha} H(x, t)=\int_{\mathbb{R}} M_{\alpha}(x, t, \xi) \mathrm{d} \xi,
$$

and

$$
X(x, t)=\int_{\mathbb{R}} \mathcal{K}(\xi) M(x, t, \xi) \mathrm{d} \xi .
$$

The proof is obtained by a simple integration in $\xi$ of the set of equations (4.83) against the matrix $\mathcal{K}(\xi)$. First, an integration in $\xi$ of (4.83) gives the continuity equation (2.11) i.e.

$$
\frac{\partial l_{\alpha} H}{\partial t}+\frac{\partial l_{\alpha} H u_{\alpha}}{\partial x}=G_{\alpha+1 / 2}-G_{\alpha-1 / 2},
$$

and by summation we have (3.70). Actually from the definition (4.81) of $N_{\alpha+1 / 2}$ we have

$$
\int_{\mathbb{R}} N_{\alpha+1 / 2}(x, t, \xi) \mathrm{d} \xi=G_{\alpha+1 / 2}(x, t),
$$

and

$$
\int_{\mathbb{R}} \xi N_{\alpha+1 / 2}(x, t, \xi) \mathrm{d} \xi=u_{\alpha+1 / 2} G_{\alpha+1 / 2} .
$$

Likewise for the energy balance of the layer $\alpha$ we proceed an integration in $\xi$ of (4.83) against $\xi^{2} / 2$. Since we have

$$
\begin{gathered}
\int_{\mathbb{R}}\left(\frac{\xi^{2}}{2} M_{\alpha}+\widetilde{M}_{\alpha}\right) \mathrm{d} \xi=\frac{h_{\alpha}}{2} u_{\alpha}^{2}+\frac{g}{2} h_{\alpha} H \\
\int_{\mathbb{R}} \xi\left(\frac{\xi^{2}}{2} M_{\alpha}+\widetilde{M}_{\alpha}\right) \mathrm{d} \xi=\frac{h_{\alpha}}{2} u_{\alpha}^{3}+g h_{\alpha} H u_{\alpha},
\end{gathered}
$$


and for the source term

$$
\begin{aligned}
\int_{\mathbb{R}} \frac{\xi^{2}}{2} \frac{\partial}{\partial x}\left(p^{a}+g z_{b}\right) \frac{\partial M_{\alpha}}{\partial \xi} \mathrm{d} \xi= & -\frac{\partial}{\partial x}\left(p^{a}+g z_{b}\right) h_{\alpha} u_{\alpha} \\
= & -\frac{\partial}{\partial x}\left(\left(g z_{b}+p^{a}\right) h_{\alpha} u_{\alpha}\right)+\left(g z_{b}+p^{a}\right) \frac{\partial h_{\alpha} u_{\alpha}}{\partial x} \\
= & -\frac{\partial}{\partial x}\left(\left(g z_{b}+p^{a}\right) h_{\alpha} u_{\alpha}\right)-\left(g z_{b}+p^{a}\right) \frac{\partial h_{\alpha}}{\partial t} \\
& -\left(g z_{b}+p^{a}\right) G_{\alpha+1 / 2}+\left(g z_{b}+p^{a}\right) G_{\alpha-1 / 2},
\end{aligned}
$$

we obtain the equality

$$
\begin{gathered}
\frac{\partial}{\partial t}\left(\frac{h_{\alpha}}{2} u_{\alpha}^{2}+\frac{g}{2} h_{\alpha}\left(\eta+z_{b}\right)+h_{\alpha} p^{a}\right)+\frac{\partial}{\partial x}\left[u_{\alpha}\left(h_{\alpha} u_{\alpha}^{2}+\frac{g}{2} h_{\alpha} H+\frac{g}{2} h_{\alpha}\left(\eta+z_{b}\right)+h_{\alpha} p^{a}\right)\right] \\
+\frac{u_{\alpha-1 / 2}^{2}}{2} G_{\alpha-1 / 2}-\frac{u_{\alpha+1 / 2}^{2}}{2} G_{\alpha+1 / 2}-h_{\alpha} \frac{\partial p^{a}}{\partial t}-g h_{\alpha} \frac{\partial z_{b}}{\partial t}=\int_{\mathbb{R}}\left(\frac{\xi^{2}}{2} Q_{\alpha}+\widetilde{Q}_{\alpha}\right) \mathrm{d} \xi .
\end{gathered}
$$

The previous relation corresponds to (4.78) where the viscous and friction terms are neglected. The sum of the equations (4.91) gives the energy equality for the global system and that completes the proof.

The formulation (4.83) reduces the nonlinear multilayer Saint-Venant system to a linear transport system on nonlinear quantities $\left\{M_{\alpha}\right\}_{\alpha=1}^{N},\left\{N_{\alpha+1 / 2}\right\}_{\alpha=0}^{N}$ for which it is easier to find a simple numerical scheme with good theoretical properties. In the case of a single layer, for a detailed proof of the kinetic interpretation refer to [3] and for the treatment of the source term at this microscopic level see [25]. Notice that the choice of the function $\chi$ remains quite open at this stage since several functions satisfy the requested properties. Following this choice the deduced kinetic scheme will have different properties.

\section{Numerical Results}

In the applications discussed here, we assume $p^{a}=0$ and we neglect the horizontal viscosity. Then the $N+1$ equations of the multilayer system (3.70)-(3.73) can be written with the general form

$$
\begin{aligned}
& \frac{\partial H}{\partial t}+\sum_{\alpha=1}^{N} \frac{\partial\left(l_{\alpha} H u_{\alpha}\right)}{\partial x}=0, \\
& \frac{\partial\left(l_{\alpha} H u_{\alpha}\right)}{\partial t}+\frac{\partial}{\partial x}\left(l_{\alpha} H u_{\alpha}^{2}+\frac{g}{2} l_{\alpha} H^{2}\right)=-g l_{\alpha} H \frac{\partial z_{b}}{\partial x}+u_{\alpha+1 / 2} G_{\alpha+1 / 2}-u_{\alpha-1 / 2} G_{\alpha-1 / 2} \\
& \quad+\frac{2 \nu_{\alpha}}{l_{\alpha+1}+l_{\alpha}} \frac{u_{\alpha+1}-u_{\alpha}}{H}-\frac{2 \nu_{\alpha-1}}{l_{\alpha}+l_{\alpha-1}} \frac{u_{\alpha}-u_{\alpha-1}}{H}-\kappa_{\alpha}(u, H) u_{\alpha}, \quad \alpha=1, \ldots, N,
\end{aligned}
$$

with

$$
\kappa_{\alpha}=\left\{\begin{array}{l}
\kappa(u, H) \text { if } \alpha=1 \\
0 \text { if } \quad \alpha \neq 1
\end{array} \quad \nu_{\alpha}=\left\{\begin{array}{lll}
0 & \text { if } & \alpha=0 \\
\nu & \text { if } & \alpha=1, \ldots, N-1 \\
0 & \text { if } & \alpha=N .
\end{array}\right.\right.
$$

The previous system is of the form:

$$
\frac{\partial X}{\partial t}+\frac{\partial F(X)}{\partial x}=S b(X)+S e(X)+S v(X)
$$

with $F(X)$ the flux of the hyperbolic part, $S b(X)$ the topography source term, $S e(X)$ the mass transfer source term and $S v(X)$ the viscous and friction terms. 
To approximate the solution of the multilayer Saint-Venant system, we use a finite volume framework. We assume that the computational domain is discretised by $I$ nodes $x_{i}$. We denote $C_{i}$ the cell of length $\Delta x_{i}=x_{i+1 / 2}-x_{i-1 / 2}$ with $x_{i+1 / 2}=\left(x_{i}+x_{i+1}\right) / 2$. For the time discretization, we denote $t^{n}=\sum_{k \leq n} \Delta t^{k}$ where the time steps $\Delta t^{k}$ will be precised later though a CFL condition. We denote $X_{i}^{n}=\left(H_{i}^{n}, q_{1, i}^{n}, \ldots, q_{N, i}^{n}\right)$ the approximate solution at time $t^{n}$ on the cell $C_{i}$ with $q_{\alpha, i}^{n}=l_{\alpha} H_{i}^{n} u_{\alpha, i}^{n}$.

\subsection{Time discretization}

For the time discretization, we apply time splitting to equation (5.94) and we write

$$
\begin{aligned}
& \frac{\tilde{X}^{n+1}-X^{n}}{\Delta t^{n}}+\frac{\partial F\left(X^{n}\right)}{\partial x}=S b\left(X^{n}\right)+S e\left(X^{n}\right), \\
& \frac{X^{n+1}-\tilde{X}^{n+1}}{\Delta t^{n}}-S v\left(X^{n}, X^{n+1}\right)=0 .
\end{aligned}
$$

Classically we first compute the hyperbolic part (5.95) of the multilayer system by an explicit scheme. This first computation includes the topographic source term in order to preserve relevant equilibria [5] and also defines the mass transfer terms. Concerning the viscous and friction terms (5.96) that are dissipative, we prefer a semi-implicit scheme for reasons of stability.

\subsection{Numerical scheme: explicit part}

To perform the explicit step we deduce a finite volume kinetic scheme from the previous kinetic interpretation of the multilayer system. Notice that even if the system is hyperbolic, the eigenvalues are unknown. Thus any solver requiring the knowledge of the eigenvalues would face difficulties while but the kinetic scheme is easily extended [4].

Starting from a piecewise constant approximation of the initial data, the general form of a finite volume method is

$$
\tilde{X}_{i}^{n+1}-X_{i}^{n}+\sigma_{i}^{n}\left[F_{i+1 / 2}^{n}-F_{i-1 / 2}^{n}\right]=\Delta t^{n} S b_{i}^{n}+\Delta t^{n} S e_{i}^{n},
$$

where $\sigma_{i}^{n}=\Delta t^{n} / \Delta x_{i}$ is the ratio between space and time steps and the numerical flux $F_{i+1 / 2}^{n}$ is an approximation of the exact flux estimated at point $x_{i+1 / 2}$.

The topographic source term $S b_{i}^{n}$ is not deduced from the kinetic interpretation (see [25]) but computed by hydrostatic reconstruction, see Proposition 5.1. As in [3,6] the kinetic interpretation (4.83) is used to precise the expression of the fluxes $F_{i+1 / 2}^{n}$ in (5.97). First, by analogy with (4.80) we define the discrete densities of particles $M_{\alpha, i}^{n}$ by

$$
M_{\alpha, i}^{n}(\xi)=l_{\alpha} \frac{H_{i}^{n}}{c_{i}^{n}} \chi\left(\frac{\xi-u_{\alpha, i}^{n}}{c_{i}^{n}}\right), \quad \text { with } c_{i}^{n}=\sqrt{\frac{g H_{i}^{n}}{2}} .
$$

Then the equation (4.83) without the atmospheric pressure and topographic terms is discretised for each $\alpha$ by applying a simple upwind scheme for the advection term

$$
f_{\alpha, i}^{n+1}(\xi)=M_{\alpha, i}^{n}(\xi)-\xi \sigma_{i}^{n}\left(M_{\alpha, i+1 / 2}^{n}(\xi)-M_{\alpha, i-1 / 2}^{n}(\xi)\right)+\Delta t^{n}\left(N_{\alpha+1 / 2, i}^{n+1 / 2}(\xi)-N_{\alpha-1 / 2, i}^{n+1 / 2}(\xi)\right),
$$

where

$$
M_{\alpha, i+1 / 2}^{n}= \begin{cases}M_{\alpha, i}^{n} & \text { if } \xi \geq 0 \\ M_{\alpha, i+1}^{n} & \text { if } \xi<0\end{cases}
$$

and the terms $N_{\alpha+1 / 2, i}^{n+1 / 2}$ will be defined in the following.

We define the vectors $f_{i}^{n+1}(\xi)=\left(f_{1, i}^{n+1}(\xi), \ldots, f_{N, i}^{n+1}(\xi)\right)^{T}, M_{i}^{n}(\xi)=\left(M_{1, i}^{n}(\xi), \ldots, M_{N, i}^{n}(\xi)\right)^{T}$. Each new density function $f_{\alpha, i}^{n+1}$ is not an equilibrium but thanks to the property of the right hand side of (4.83), by analogy 
with (4.86), (4.87) we can recover the macroscopic quantities at time $t^{n+1}$. We write

$$
l_{\alpha} H_{i}^{n+1}=\int_{\mathbb{R}} f_{i}^{n+1}(\xi) \mathrm{d} \xi
$$

and by a simple integration in $\xi$ of (5.98) against $\mathcal{K}(\xi)$, we can precise the macroscopic formula (5.97) (without the topographic term)

$$
\tilde{X}_{i}^{n+1}=\int_{\mathbb{R}} \mathcal{K}(\xi) f_{i}^{n+1}(\xi) \mathrm{d} \xi
$$

If we denote

$$
F_{i+1 / 2}^{n}=F\left(X_{i}^{n}, X_{i+1}^{n}\right)=F^{+}\left(X_{i}^{n}\right)+F^{-}\left(X_{i+1}^{n}\right),
$$

we define

$$
F^{-}\left(X_{i}^{n}\right)=\int_{\xi \in \mathbb{R}^{-}} \xi \mathcal{K}(\xi) M_{i}^{n}(\xi) \mathrm{d} \xi, \quad F^{+}\left(X_{i}^{n}\right)=\int_{\xi \in \mathbb{R}^{+}} \xi \mathcal{K}(\xi) M_{i}^{n}(\xi) \mathrm{d} \xi .
$$

More precisely the expression of $F^{+}\left(X_{i}\right)$ can be written

$$
F^{+}\left(X_{i}\right)=\left(\begin{array}{c}
F_{H}^{+}\left(X_{i}\right) \\
F_{q_{1}}^{+}\left(X_{i}\right) \\
\vdots \\
F_{q_{N}}^{+}\left(X_{i}\right)
\end{array}\right),
$$

with

$$
\begin{aligned}
& F_{H}^{+}\left(X_{i}\right)=\sum_{\alpha=1}^{N} F_{h_{\alpha}}^{+}\left(X_{i}\right)=\sum_{\alpha=1}^{N} l_{\alpha} H \int_{w \geq-\frac{u_{\alpha, i}}{c_{i}}}\left(u_{\alpha, i}+w c_{i}\right) \chi(w) \mathrm{d} w \\
& F_{q_{\alpha}}^{+}\left(X_{i}\right)=l_{\alpha} H \int_{w \geq-\frac{u_{\alpha, i}}{c_{i}}}\left(u_{\alpha, i}+w c_{i}\right)^{2} \chi(w) \mathrm{d} w .
\end{aligned}
$$

We denote also

$$
\mathcal{F}_{h_{\alpha}, i}=F_{h_{\alpha}, i+1 / 2}-F_{h_{\alpha}, i-1 / 2}=F_{h_{\alpha}}^{+}\left(X_{i}\right)+F_{h_{\alpha}}^{-}\left(X_{i+1}\right)-\left(F_{h_{\alpha}}^{+}\left(X_{i-1}\right)+F_{h_{\alpha}}^{-}\left(X_{i}\right)\right) .
$$

This kinetic method is interesting because it gives a very simple and natural way to propose a numerical flux through the kinetic interpretation. If we can perform analytically the integration in (5.102), i.e. if the probability function $\chi$ defined in (4.79) is chosen to be simple enough, it is also numerically powerful because the kinetic level disappears and the scheme is written directly as a macroscopic scheme for which only very simple computations are needed. In this paper we have used

$$
\chi(w)=\frac{1}{2 \sqrt{3}} 1_{|w| \leq \sqrt{3}}(w) .
$$

Let us now precise the terms $N_{\alpha+1 / 2, i}^{n+1 / 2}$ and so the exchange terms $S e_{i}^{n}$ defined by

$$
S e_{i}^{n}=\int_{\mathbb{R}} \mathcal{K}(\xi)\left(N_{\alpha+1 / 2, i}^{n+1 / 2}(\xi)-N_{\alpha-1 / 2, i}^{n+1 / 2}(\xi)\right) \mathrm{d} \xi
$$

From the conditions (4.82) we prescribe

$$
N_{1 / 2, i}^{n+1 / 2}(\xi)=N_{N+1 / 2, i}^{n+1 / 2}(\xi)=0 .
$$


So we recover $S e_{H, i}^{n}=0$ and equation (5.100) defines $H_{i}^{n+1}$. By summation of (5.98) we have

$$
\Delta t^{n} N_{\alpha+1 / 2, i}^{n+1 / 2}(\xi)=\sum_{j=1}^{\alpha}\left(f_{j, i}^{n+1}(\xi)-M_{j, i}^{n}(\xi)+\xi \sigma_{i}^{n}\left(M_{j, i+1 / 2}^{n}(\xi)-M_{j, i-1 / 2}^{n}(\xi)\right)\right), \quad \alpha=1, \ldots, N-1,
$$

and we define

$$
G_{\alpha+1 / 2, i}^{n+1 / 2}=\int_{\mathbb{R}} N_{\alpha+1 / 2, i}^{n+1 / 2}(\xi) \mathrm{d} \xi, \quad \alpha=0, \ldots, N
$$

so we can write

$$
\Delta t^{n} G_{\alpha+1 / 2, i}^{n+1 / 2}=\sum_{j=1}^{\alpha}\left[l_{j}\left(H_{i}^{n+1}-H_{i}^{n}\right)+\sigma_{i}^{n}\left(F_{h j, i+1 / 2}^{n}-F_{h j, i-1 / 2}^{n}\right)\right], \quad \alpha=1, \ldots, N .
$$

Then using the discrete mass conservation equation giving $H_{i}^{n+1}$, the terms $G_{\alpha+1 / 2, i}^{n+1 / 2}$ can be written under an explicit form (see (2.22)) i.e. depending only of $X_{i}^{n}$

$$
\Delta x_{i} G_{\alpha+1 / 2, i}^{n+1 / 2}=\sum_{j=1}^{\alpha}\left(\mathcal{F}_{h_{j}, i}^{n}-l_{j} \sum_{p=1}^{N} \mathcal{F}_{h_{p}, i}^{n}\right),
$$

we have to notice that this definition is compatible with the free surface condition of (5.105).

We define

with, according to $(2.23)$

$$
N_{\alpha+1 / 2, i}^{n+1 / 2}(\xi)=G_{\alpha+1 / 2, i}^{n+1 / 2} \delta\left(\xi-u_{\alpha+1 / 2, i}^{n}\right)
$$

$$
u_{\alpha+1 / 2, i}^{n}= \begin{cases}u_{\alpha+1, i}^{n} & \text { if } G_{\alpha+1 / 2, i}^{n+1 / 2} \geq 0, \\ u_{\alpha, i}^{n} & \text { if } G_{\alpha+1 / 2, i}^{n+1 / 2}<0 .\end{cases}
$$

Then the exchange term $S e_{i}^{n}$ in (5.104) is completely defined.

We have denoted the approximations in time of $N_{\alpha+1 / 2}$ and $G_{\alpha+1 / 2}$ with an upperscript $n+1 / 2$ because we have to define $H_{i}^{n+1}$ at the macroscopic level to obtain the microscopic approximation of $N_{\alpha+1 / 2, i}$ which is used for the computation of the momentum $l_{\alpha} H_{i}^{n+1} u_{\alpha, i}^{n+1}$.

The source term $S b_{i}^{n}=\left(S b_{H, i}^{n}, S b_{1, i}^{n}, \ldots, S b_{N, i}^{n}\right)$ is an approximation of the topographic source terms. For stability purpose, see [5] we use the following discretization

$$
S b_{H, i}^{n}=0, \quad S b_{\alpha, i}^{n}=l_{\alpha}\left(\frac{g}{2}\left(H_{i+1 / 2-}^{n}\right)^{2}-\frac{g}{2}\left(H_{i-1 / 2+}^{n}\right)^{2}\right)
$$

with

$$
\begin{aligned}
z_{b, i+1 / 2} & =\max \left\{z_{b, i}, z_{b, i+1}\right\}, \\
H_{i+1 / 2-}^{n} & =H_{i}^{n}+z_{b, i}-z_{b, i+1 / 2}, \\
H_{i+1 / 2+}^{n} & =H_{i+1}^{n}+z_{b, i+1}-z_{b, i+1 / 2} .
\end{aligned}
$$

And we have the following proposition.

Proposition 5.1. The discretization of the source terms given by (5.111), (5.112) preserves the steady states

$$
\left\{u_{\alpha, i}^{n}=0\right\}_{\alpha=1}^{N}, \quad H_{i}^{n}+z_{b, i}=\text { Cst. } \in \mathbb{R}, \quad \forall i, \quad \forall n,
$$

given by a "lake at rest". 
Proof. For the proof of this proposition, the readers can refer to [5].

The scheme explained in this paragraph allows to calculate $\tilde{X}^{n+1}$ given by (5.95) and (5.97).

\subsection{Numerical scheme: implicit part}

Now we aim to calculate $X^{n+1}$ from (5.96). Neglecting the horizontal viscosity, the vertical viscosity source term can be interpreted as a friction term between one layer and the two adjacent ones. As usual we treat this friction term implicitly. This leads to solve a linear system.

The implicit step does not affect the discrete water height therefore

$$
H_{i}^{n+1}=\tilde{H}_{i}^{n+1},
$$

and the computation of the new velocities $\left\{u_{\alpha, i}^{n+1}\right\}_{\alpha=1}^{N}$ leads to solve a tridiagonal $N \times N$ linear system that reads

$$
T_{i}^{n, n+1} U_{i}^{n+1}=\tilde{q}_{i}^{n+1},
$$

with $U_{i}^{n+1}=\left(u_{1, i}^{n+1}, \ldots, u_{N, i}^{n+1}\right)^{T}, \tilde{q}_{i}^{n+1}=\left(\tilde{q}_{1, i}^{n+1}, \ldots, \tilde{q}_{N, i}^{n+1}\right)^{T}$ and

$$
\begin{array}{ll}
T_{i}^{n, n+1}(1,1) & =l_{1} H_{i}^{n+1}+\frac{2 \Delta t^{n}}{H_{i}^{n+1}}\left(\frac{\nu_{1}}{l_{1}+l_{2}}\right)+\Delta t^{n} \kappa\left(X_{i}^{n}, H_{i}^{n+1}\right), \\
T_{i}^{n, n+1}(\alpha, \alpha) & =l_{\alpha} H_{i}^{n+1}+\frac{2 \Delta t^{n}}{H_{i}^{n+1}}\left(\frac{\nu_{\alpha}}{l_{\alpha}+l_{\alpha+1}}+\frac{\nu_{\alpha-1}}{l_{\alpha}+l_{\alpha-1}}\right), \quad \text { for } \alpha \in\{2, \ldots, N\}, \\
T_{i}^{n, n+1}(\alpha, \alpha+1) & =-\frac{2 \Delta t^{n}}{H_{i}^{n+1}}\left(\frac{\nu_{\alpha}}{l_{\alpha}+l_{\alpha+1}}\right), \quad \text { for } \alpha \in\{1, \ldots, N-1\}, \\
T_{i}^{n, n+1}(\alpha-1, \alpha) & =-\frac{2 \Delta t^{n}}{H_{i}^{n+1}}\left(\frac{\nu_{\alpha-1}}{l_{\alpha}+l_{\alpha-1}}\right), \text { for } \alpha \in\{2, \ldots, N\} .
\end{array}
$$

For the friction at the bottom, several models can be used among which are Navier, Chezy and Strickler laws.

\subsection{Stability of the scheme}

We now establish the stability property of the kinetic scheme. Classically for the Saint-Venant system, a CFL condition ensures the water height is non negative. This CFL condition means that the quantity of water leaving a given cell during a time step $\Delta t^{n}$ is less than the actual water in the cell.

For the multilayer Saint-Venant system we have the same kind of requirement concerning the time step $\Delta t^{n}$. But due to the vertical discretization, the water can leave the cell $C_{i}$ of the layer $\alpha$ either by the boundaries $x_{i \pm 1 / 2}$ or by the interfaces $z_{\alpha \pm 1 / 2}$, see Figure 3 . This makes the CFL condition more restrictive and we have the following proposition.

Proposition 5.2. Assume that the function $\chi$ has a compact support of length $2 w_{M}$ then under the CFL condition

$$
\Delta t^{n} \leq \min _{1 \leq \alpha \leq N} \min _{i \in I} \frac{l_{\alpha} H_{i}^{n} \Delta x_{i}}{l_{\alpha} H_{i}^{n}\left(\left|u_{\alpha, i}^{n}\right|+w_{M} c_{i}^{n}\right)+\Delta x_{i}\left(\left[G_{\alpha+1 / 2, i}^{n+1 / 2}\right]_{-}+\left[G_{\alpha-1 / 2, i}^{n+1 / 2}\right]_{+}\right)}
$$

the kinetic scheme (5.97), (5.111) and (5.102) keeps the water height positive i.e. $H_{i}^{n} \geq 0$ if it is true initially. Notice that this condition does not depend on $\frac{\partial z_{b}}{\partial x}$.

Proof. The proof has been adapted from those given in $[2,25]$. To prove the stability property of the scheme, we come back to the kinetic interpretation and we proceed by induction. We assume that $H_{i}^{n} \geq 0, \forall i$ and we prove that $H_{i}^{n+1} \geq 0, \forall i$. 


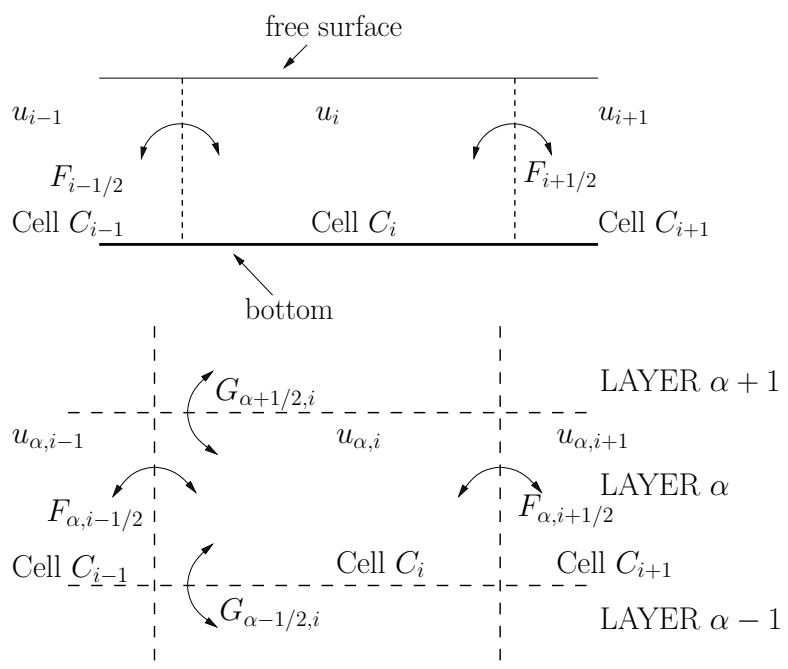

Figure 3. Interpretation of the CFL condition for the classical Saint-Venant system (up) and for the multilayer system (down).

From the definition of the functions $M_{\alpha}$ in (4.80) and the positivity of the function $\chi$, we deduce

$$
M_{\alpha, i}^{n} \geq 0, \quad \forall i, \quad \text { for } \alpha=1, \ldots, N \text {. }
$$

We now introduce the quantities

$$
[\xi]_{+}=\max (0, \xi), \quad[\xi]_{-}=\max (0,-\xi)
$$

and so we can write the upwind microscopic scheme (5.98)

$$
\begin{aligned}
f_{\alpha, i}^{n+1}= & \left(1-\sigma_{i}^{n}|\xi|\right) M_{\alpha, i}^{n}+\sigma_{i}^{n}[\xi]_{+} M_{\alpha, i-1}^{n}+\sigma_{i}^{n}[\xi]_{-} M_{\alpha, i+1}^{n}+\Delta t^{n}\left(\left(\left[N_{\alpha+1 / 2, i}^{n+1 / 2}\right]_{+}-\left[N_{\alpha+1 / 2, i}^{n+1 / 2}\right]_{-}\right)\right. \\
& \left.-\left(\left[N_{\alpha-1 / 2, i}^{n+1 / 2}\right]_{+}-\left[N_{\alpha-1 / 2, i}^{n+1 / 2}\right]_{-}\right)\right)
\end{aligned}
$$

The quantity

$$
\sigma_{i}^{n}|\xi| M_{\alpha, j}^{n}+\Delta t^{n}\left(\left[N_{\alpha+1 / 2, i}^{n+1 / 2}\right]_{-}+\left[N_{\alpha-1 / 2, i}^{n+1 / 2}\right]_{+}\right)
$$

represents, at the microscopic level, the water leaving the cell $C_{i}$ of the layer $\alpha$ during $\Delta t^{n}$. A sufficient condition to obtain the stability property, i.e.

$$
l_{\alpha} H_{i}^{n+1}=\int_{\mathbb{R}} f_{\alpha, i}^{n+1} \mathrm{~d} \xi \geq 0, \quad \forall i, \quad \text { for } \alpha=1, \ldots, N
$$

is then

$$
\int_{\mathbb{R}}\left(\sigma_{i}^{n}|\xi| M_{\alpha, i}^{n}+\Delta t^{n}\left(\left[N_{\alpha+1 / 2, i}^{n+1 / 2}\right]_{-}+\left[N_{\alpha-1 / 2, i}^{n+1 / 2}\right]_{+}\right)\right) \mathrm{d} \xi \leq \int_{\mathbb{R}} M_{\alpha, i}^{n} \mathrm{~d} \xi,
$$

and this requirement is satisfied when

$$
\sigma_{i}^{n}\left(\left|u_{\alpha, i}^{n}\right|+w_{M} c_{i}^{n}\right) l_{\alpha} H_{i}^{n}+\Delta t^{n}\left(\left[G_{\alpha+1 / 2, i}^{n+1 / 2}\right]_{-}+\left[G_{\alpha-1 / 2, i}^{n+1 / 2}\right]_{+}\right) \leq l_{\alpha} H_{i}^{n}
$$




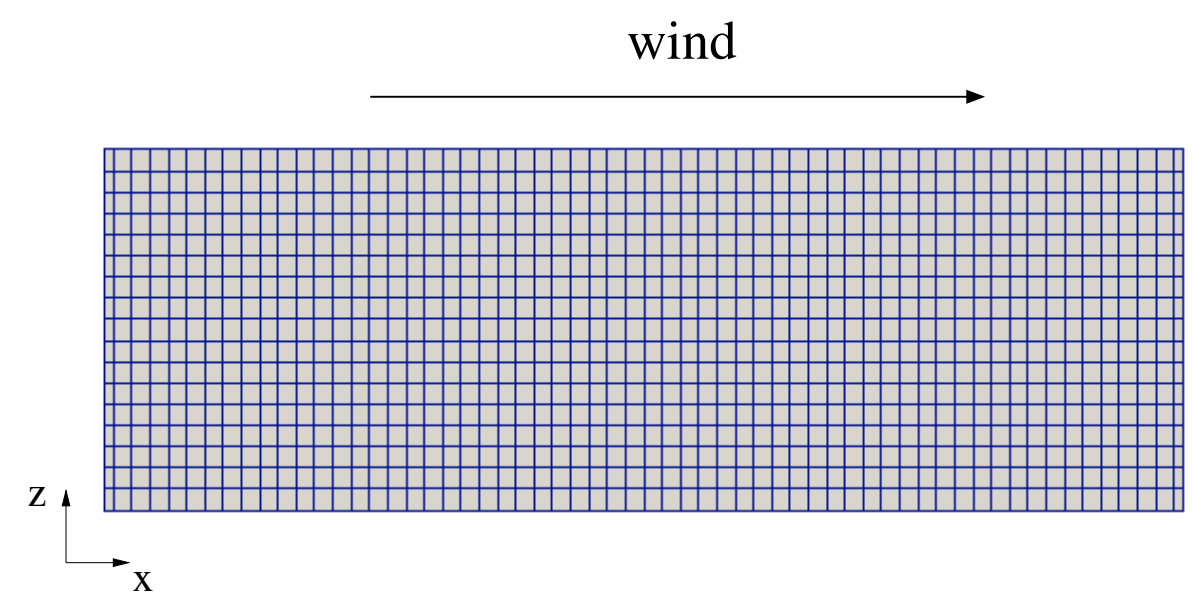

FIgURE 4. The geometrical model with the horizontal mesh and the vertical discretization by layers. In the horizontal direction, only 60 nodes are shown.

We recall that we have obtained in (5.109) an explicit form of $G_{\alpha+1 / 2, i}^{n+1 / 2}$. If $\Delta t^{n}$ satisfies (5.113), then the condition (5.115) is satisfied and that completes the proof.

\subsection{Second order scheme}

The second-order accuracy in time is usually recovered by the Heun method [8] that is a slight modification of the second order Runge-Kutta method. The advantage of the Heun scheme is that it preserves the invariant domains without any additional limitation on the CFL.

We also apply a formally second order scheme in space by a limited reconstruction of the variables. An advantage of the new multilayer approach with only one continuity equation is that the water height can be reconstructed while preserving the mass conservation without difficulty.

\subsection{Numerical simulations}

In this paragraph we give several validations of the numerical scheme previously described. We first consider a wind driven flow where a local analytical solution is available. Then we use the model in the classical test case of a supercritical regime with important variations of the free surface. Finally we compare the multilayer model with a 2D- $(x, z)$ hydrostatic Navier-Stokes system based on a finite elements discretization and developed in FreeFem $++[17]$.

\subsubsection{Analytical solution}

We claim in the introduction that the great interest of the new multilayer formulation that we proposed here is to allow mass exchanges between layers. This effect is exhibited in the numerical test that we present now. We consider a fluid in a rectangular closed basin with vertical shores and we impose a constant wind stress (from left to right) at the free surface. The flow is then supposed to reach a stationary state with a recirculation in the lake. Notice that this kind of stationary flows is clearly impossible to compute with the classical one-layer shallow water system since the velocity is imposed to be constant along the vertical. They are also out of the domain of application of the former multilayer shallow water system that was introduced by Audusse [1] since they clearly involve large mass transfers (at least near the shores) between the layers. It is easy to deduce from the mass conservation equations that the only stationary regime of the former multilayer model for a flow in a closed basin is the flow at rest, as for the classical Saint-Venant model. The need of the new approach becomes here very clear. 


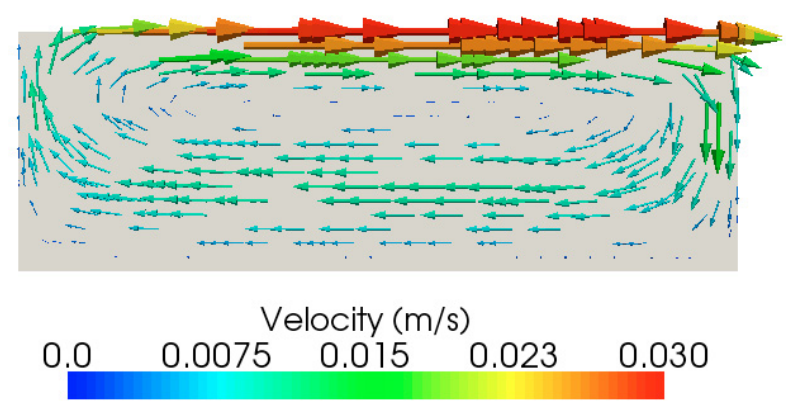

Figure 5. The wind blows from the left part of the domain to the right part. The arrows represent the velocity field in the lake.

The domain is $16 \mathrm{~m}$ long with an initial water height of $2 \mathrm{~m}$. The mesh has 160 nodes in the $x$ direction and 20 layers. The wind velocity (from left to right) is $20 \mathrm{~m} \cdot \mathrm{s}^{-1}$. We show the results on a postprocessing $2 \mathrm{D}$ mesh that is presented in Figure 4. In Figure 5 we also present the two dimensional velocity vectors on this $2 \mathrm{D}$ mesh. The results exhibit a global recirculation. Since we consider a shallow water type system, the vertical velocity is not a variable of the computation. But it is possible to recover it for postprocessing purpose: departing from the computed horizontal velocity we integrate the divergence free condition (3.31) on each layer and we use the non penetration condition at the bottom (2.5) to evaluate an approximation of the vertical velocity.

For this test case, the bottom velocity is imposed to be zero by prescribing a very large Navier friction coefficient. Then there exists an analytical solution of the velocity profile at mid-length of the lake. This calculation is detailed in [27]. In Figure 6a we compare the analytical solution with the results obtained with the multilayer model for different vertical discretizations. We have used a viscosity of $\nu=0.01 \mathrm{~m}^{2} \cdot \mathrm{s}^{-1}$. In Figure $6 \mathrm{~b}$, we plot the rate of error versus the vertical discretization, namely the number of layers. We have plotted the $\log \left(L^{1}\right.$ - error $)$ of the horizontal velocity - at mid-length of the domain - versus $\log \left(h_{0} / h_{i}\right)$. We denote by $h_{i}$ the average cell height, $h_{0}$ is the average cell height of the coarser mesh. These errors have been computed on 4 meshes with 5, 10, 20 and 30 layers. For each mesh, the horizontal discretization is taken very precise meaning the error associated with the horizontal discretization can be neglected. Even if we use a second order scheme (see Sect. 5.5) for the hyperbolic part of the model, the friction and exchange terms are discretized using a 1st order scheme. So it appears that the computed convergence rates are closed to 1.7.

\subsubsection{Transcritical flow over a bump}

Then we consider an academic test case that is very commonly used for the validation of classical one-layer shallow water solvers. Here we add some friction at the bottom in order to compare solutions of one-layer and multilayer shallow water systems with the solution of hydrostatic incompressible Navier-Stokes equations. We impose an inflow (left boundary) of $1.0 \mathrm{~m}^{2} \cdot \mathrm{s}^{-1}$ and the water height at the exit (right boundary) is prescribed to be equal to $0.6 \mathrm{~m}$. The Strickler friction coefficient at the bottom is $30 \mathrm{~m}^{-3} \cdot \mathrm{s}^{-1}$ and the kinematic viscosity is $0.01 \mathrm{~m}^{2} \cdot \mathrm{s}^{-1}$. The data are chosen such that the flow is supposed to reach a stationary regime that presents some transitions between sub- and supercritical parts and an hydraulic jump. Notice that an analytical solution exists for this test in the case of a single layer [3,25].

The simulation results are depicted in Figures 7-9. Notice that the actual computations are purely one dimensional. Hence Figures 7 and 8 present velocity results on a postprocessing mesh that is constructed departing from the $1 \mathrm{~d}$ mesh by the use of the computed layer water heights. The presented results correspond to an instant $t_{f}$ where the permanent regime is achieved.

The results depicted in Figures 7 and 8 are consistent with computations performed using the hydrostatic Navier-Stokes equations of TELEMAC-3d [6,14] and also using the former multilayer Saint-Venant system [6]. The results depicted in Figure 9 exhibit that the presented solver is quite robust since it is able to compute transcritical solutions and shock waves even when a large number of layers is considered. This kind of robustness 


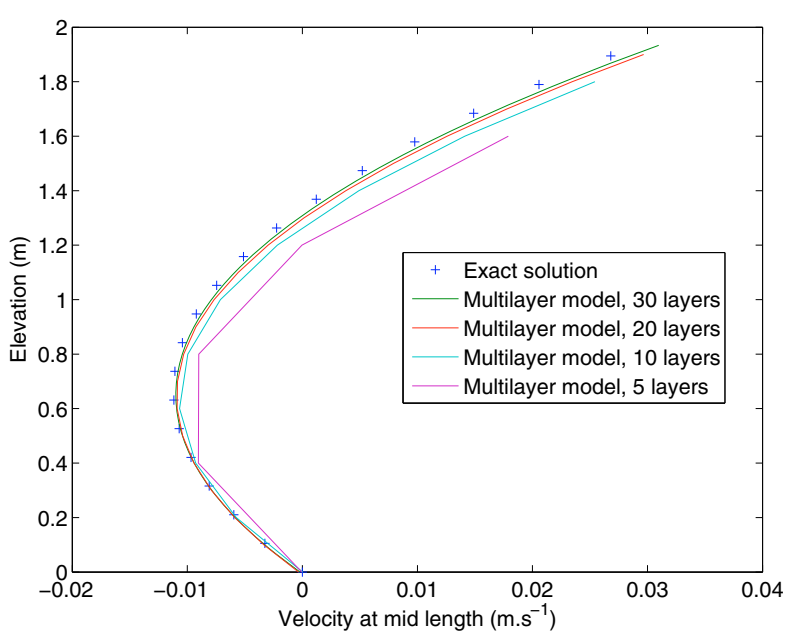

(a)

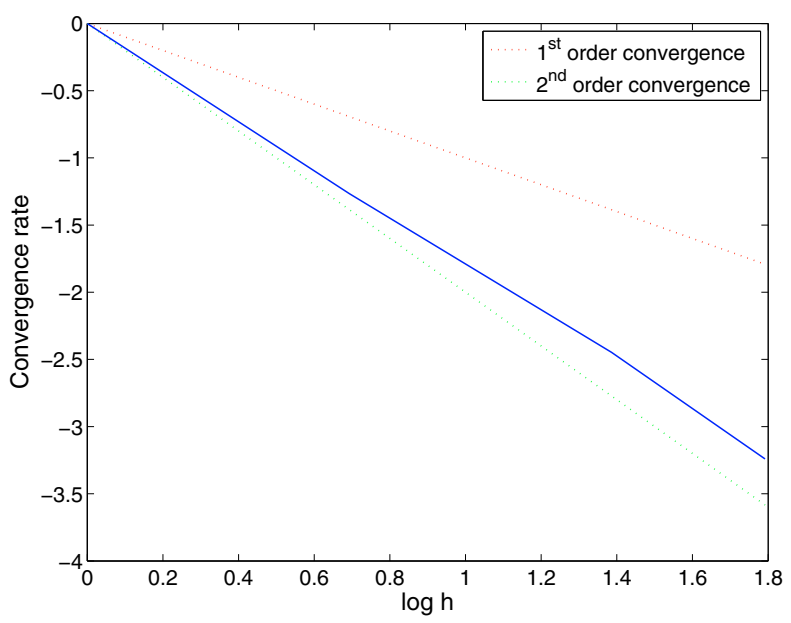

(b)

FiguRE 6. (a) Comparison between the analytical and simulated solutions and (b) convergence rate.

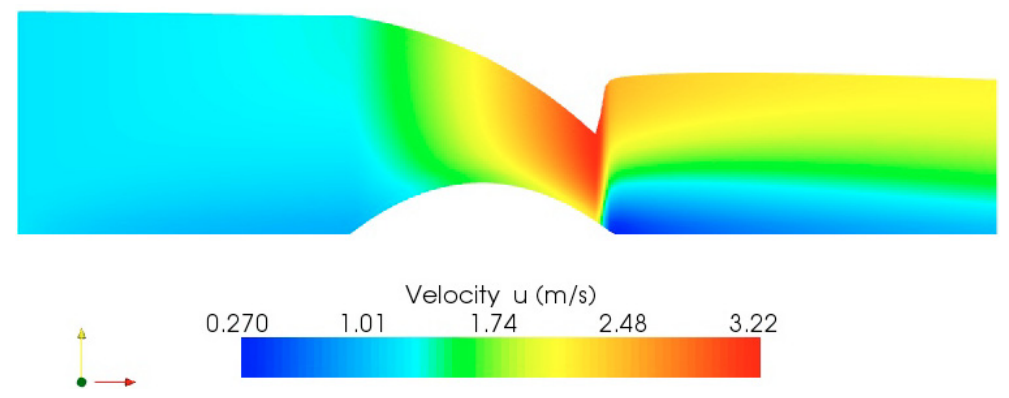

FiguRE 7. Horizontal velocities $\left\{u_{\alpha}\left(x, t_{f}\right)\right\}_{\alpha=1}^{N}$ with $N=15$ layers.

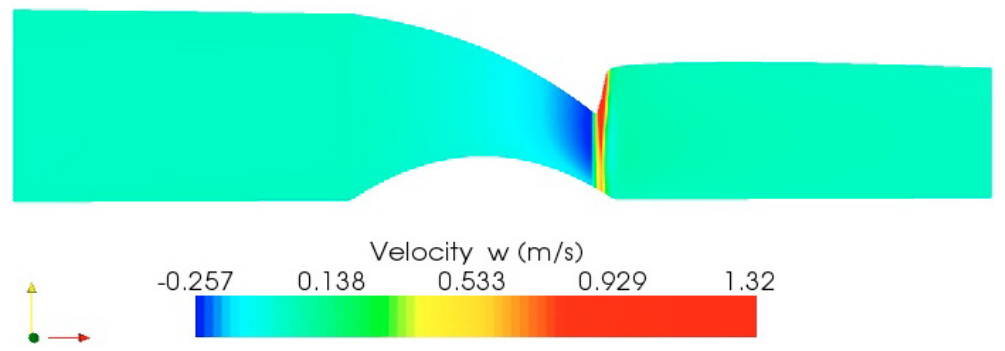

Figure 8. Vertical velocity $\left\{w_{\alpha}\left(x, t_{f}\right)\right\}_{\alpha=1}^{N}$ with $N=15$ layers.

is far from being obvious for 3d Navier-Stokes solvers where the mesh has to follow the movements of the free surface. Notice also that the hydraulic jump appears to be overestimated by the one-layer computation when compared with other results - see Figure 9. 


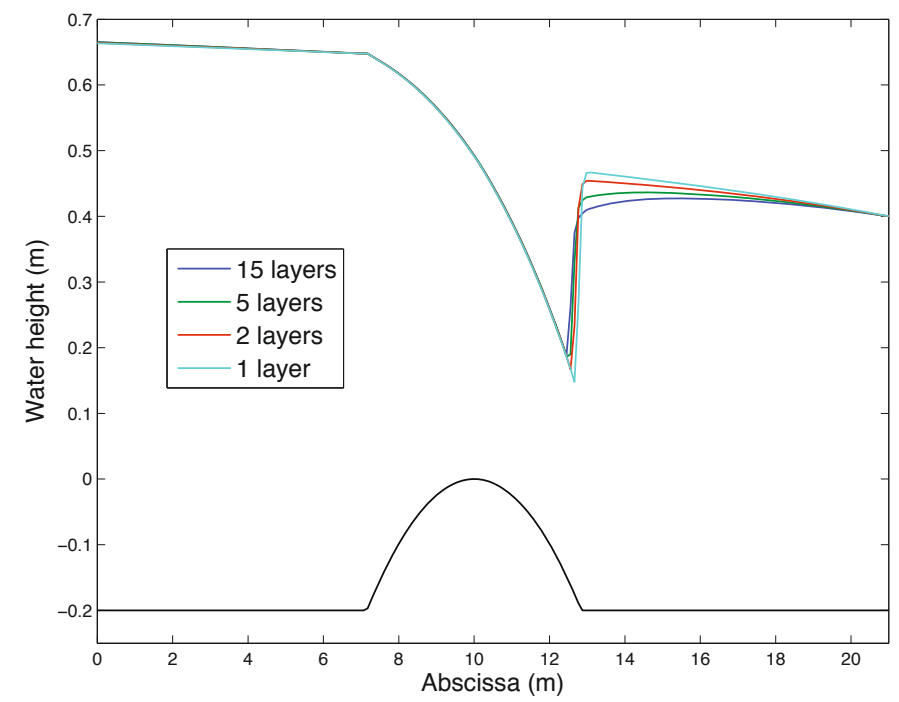

FigURE 9. Shape of the free surface for simulations carried out with different number of layers.

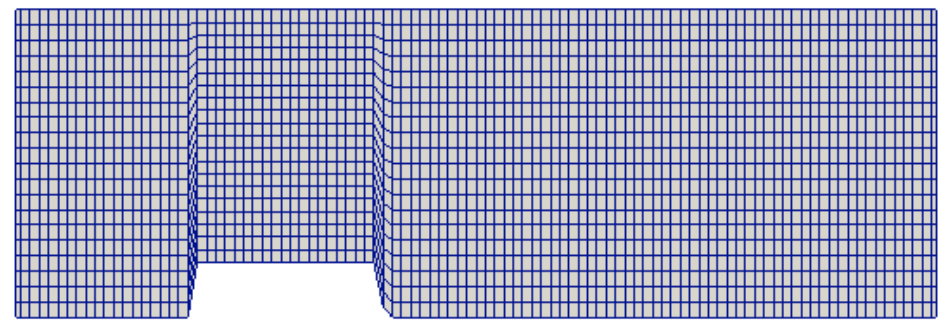

FiguRE 10. The geometrical model with the horizontal mesh and the vertical discretization by layers.

\subsubsection{Comparison with a finite element simulations of the hydrostatic Navier-Stokes system}

We have also compared our simulations with results obtained using a finite element discretization of the hydrostatic Navier-Stokes system. The finite element formulation is $P_{1} / P_{1}$ for the velocity and the pressure and uses a stabilization technique. The formulation is available in the FreeFem ++ code [17].

We still consider a wind driven flow with vertical shores but with a non trivial bottom. The domain is $6 \mathrm{~m}$ long with an initial water height of $2 \mathrm{~m}$. The mesh has 160 nodes in the $x$ direction and 20 layers. The wind velocity (from left to right) is $10 \mathrm{~m} \cdot \mathrm{s}^{-1}$. We have used a viscosity of $\nu=0.1 \mathrm{~m}^{2} \cdot \mathrm{s}^{-1}$ and a Navier type bottom friction with $\kappa=0.1 \mathrm{~m} \cdot \mathrm{s}^{-1}$. For each simulation (multilayer approach or finite elements approximation), the mesh has approximatively 1000 nodes. For the multilayer approach, the results on a postprocessing 2D mesh (see Fig. 10) are presented in Figure 11 where we have plotted the two dimensional velocity vectors $(u, w)^{T}$. In Figure 12 the results obtained with the finite elements code are shown. The results exhibit a global recirculation that is combined with two local recirculations that are induced by the topography of the lake. The qualitative aspect of the solution is consistent with the previsions.

The FreeFem ++ code, solving the full Navier-Stokes system serves as a reference. It is however not straightforward to analyze the origin of the differences because the boundary conditions are not imposed in the same way. But in the examples presented in Figures 11 and 12 whereas the viscosity and the bottom friction are 


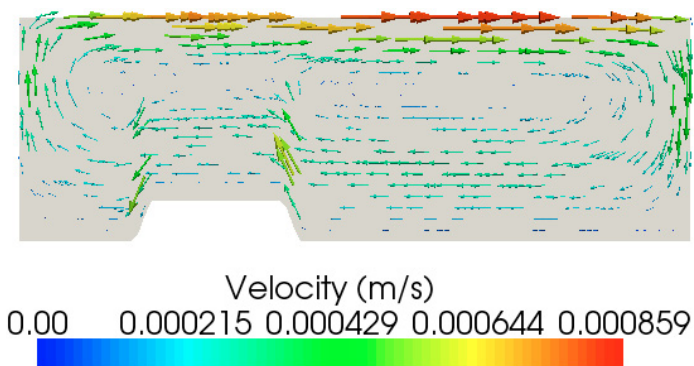

(a)

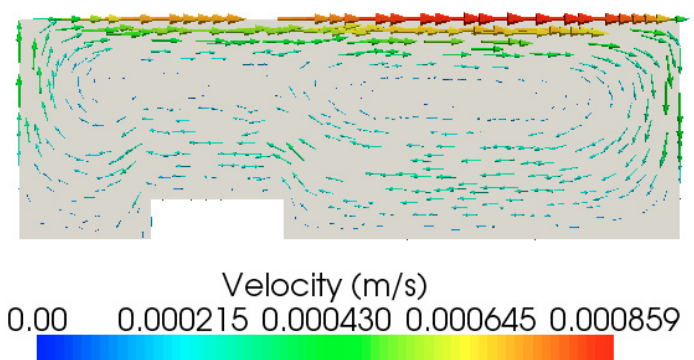

(b)

FiguRE 11. The wind blows from the left part of the domain to the right part. The arrows represent the velocity field in the lake. (a) Multilayer code and (b) finite element code.

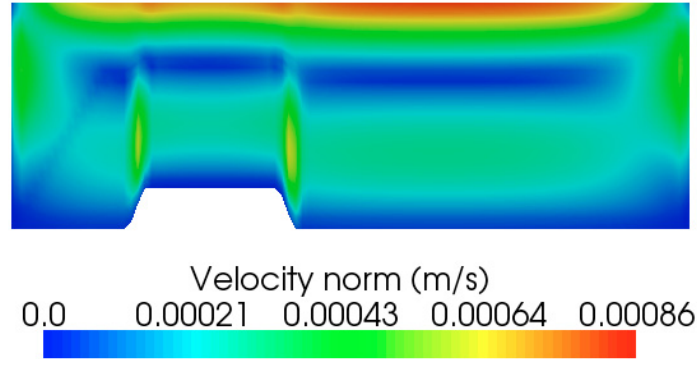

(a)

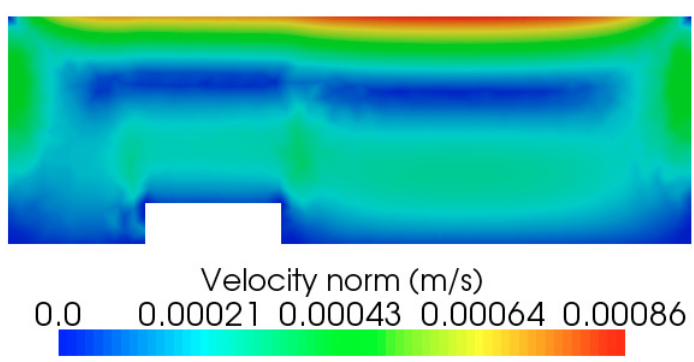

(b)

Figure 12. Same as Figure 11 but we plot the velocity norm $\sqrt{u^{2}+w^{2}}$.

large, the simulations are in agreement. The vertical velocity $w$ is overestimated by the multilayer code. This mainly comes from the fact that in the multilayer approach, $w$ is calculated offline using $u$ and the divergence free condition whereas in the finite element formulation, $w$ follows a dynamical equation

$$
\tilde{\epsilon}\left(\frac{\partial w}{\partial t}+u \frac{\partial w}{\partial x}+w \frac{\partial w}{\partial z}\right)+\frac{\partial p}{\partial z}=-g+\tilde{\epsilon} \frac{\partial \Sigma_{z x}}{\partial x}+\tilde{\epsilon} \frac{\partial \Sigma_{z z}}{\partial z}
$$

with $\tilde{\epsilon} \ll 1$. Notice also that the difference of shape for the step in Figures 11 and 12 only comes from the mesh reconstruction/interpolation procedure in the multilayer approach.

It is also difficult to compare the computational costs of the multilayer model and of the FE discretization since the simulations uses different tools. Nevertheless, if the simulation cost of a single layer Saint-Venant system is $T$, it is worth being noticed that without any parallelisation, the computational cost of a $N$ layers system is only $N T$.

\section{Conclusion}

In this paper, the authors have described a multilayer Saint-Venant system with mass exchanges. The derivation of the model, the study of its main properties and a numerical scheme for its discretization are given. Because of its accuracy and simplicity, the kinetic scheme seems well adapted for the simulations of such a model. Moreover since the eigenvalues of the hyperbolic system are not explicitly known, a lot of finite volume schemes fails in this situation [4].

Some validations of the numerical scheme are also presented especially comparisons with analytical solutions and with a finite element discretization of the Navier-Stokes system. The simulations prove the robustness 
of the multilayer approach to treat as well flows with large variations of the free surface as flows with important mass exchanges. The proposed multilayer formulation approximates the hydrostatic Navier-Stokes system and all the test cases considered prove the efficiency of the approach to simulate hydrostatic free surface flows. Notice that the model and the results presented here in $2 \mathrm{~d}(x, z)$ are also available in $3 \mathrm{~d}(x, y, z)$ that allows us to solve realistic problems.

As depicted in Figure 10, the vertical discretization proposed for water height leads to a regular mesh. A strategy of "mesh refinement" based on a inhomogeneous number of layers has to be added. The presented system can be enriched in several ways. First, the hydrostatic assumption concerning the pressure terms can be relaxed leading to the models presented in [11]. Then we can also consider a passive pollutant in the flow. This implies to add a conservation equation for the pollutant concentration. Finally, we can consider the density of the fluid varies with the concentration of pollutant. These three improvements have been added to their model by the authors and will be presented in forthcoming papers.

\section{REFERENCES}

[1] E. Audusse, A multilayer Saint-Venant system: Derivation and numerical validation. Discrete Contin. Dyn. Syst. Ser. B 5 (2005) 189-214.

[2] E. Audusse and M.O. Bristeau, Transport of pollutant in shallow water flows: A two time steps kinetic method. ESAIM: M2AN 37 (2003) 389-416.

[3] E. Audusse and M.O. Bristeau, A well-balanced positivity preserving second-order scheme for shallow water flows on unstructured meshes. J. Comput. Phys. 206 (2005) 311-333.

[4] E. Audusse and M.O. Bristeau, Finite-volume solvers for a multilayer Saint-Venant system. Int. J. Appl. Math. Comput. Sci. 17 (2007) 311-319.

[5] E. Audusse, F. Bouchut, M.O. Bristeau, R. Klein and B. Perthame, A fast and stable well-balanced scheme with hydrostatic reconstruction for Shallow Water flows. SIAM J. Sci. Comput. 25 (2004) 2050-2065.

[6] E. Audusse, M.O. Bristeau and A. Decoene, Numerical simulations of 3d free surface flows by a multilayer Saint-Venant model. Int. J. Numer. Methods Fluids 56 (2008) 331-350.

[7] A.J.C. Barré de Saint-Venant, Théorie du mouvement non permanent des eaux avec applications aux crues des rivières et à l'introduction des marées dans leur lit. C. R. Acad. Sci. Paris 73 (1871) 147-154.

[8] F. Bouchut, An introduction to finite volume methods for hyperbolic conservation laws. ESAIM: Proc. 15 (2004) $107-127$.

[9] F. Bouchut and T. Morales de Luna, An entropy satisfying scheme for two-layer shallow water equations with uncoupled treatment. ESAIM: M2AN 42 (2008) 683-698.

[10] F. Bouchut and M. Westdickenberg, Gravity driven shallow water models for arbitrary topography. Commun. Math. Sci. 2 (2004) 359-389.

[11] M.O. Bristeau and J. Sainte-Marie, Derivation of a non-hydrostatic shallow water model; Comparison with Saint-Venant and Boussinesq systems. Discrete Contin. Dyn. Syst. Ser. B 10 (2008) 733-759.

[12] M.J. Castro, J.A. García-Rodríguez, J.M. González-Vida, J. Macías, C. Parés and M.E. Vázquez-Cendón, Numerical simulation of two-layer shallow water flows through channels with irregular geometry. J. Comput. Phys. 195 (2004) 202-235.

[13] M.J. Castro, J. Macías and C. Parés, A q-scheme for a class of systems of coupled conservation laws with source term. application to a two-layer 1-D shallow water system. ESAIM: M2AN 35 (2001) 107-127.

[14] A. Decoene and J.-F. Gerbeau, Sigma transformation and ALE formulation for three-dimensional free surface flows. Int. J. Numer. Methods Fluids 59 (2009) 357-386.

[15] A. Decoene, L. Bonaventura, E. Miglio and F. Saleri, Asymptotic derivation of the section-averaged shallow water equations for river hydraulics. M3AS 19 (2009) 387-417.

[16] S. Ferrari and F. Saleri, A new two-dimensional Shallow Water model including pressure effects and slow varying bottom topography. ESAIM: M2AN 38 (2004) 211-234.

[17] FreeFem++ home page, http://www.freefem.org/ff++/index.htm (2009).

[18] J.-F. Gerbeau and B. Perthame, Derivation of viscous Saint-Venant system for laminar shallow water; Numerical validation. Discrete Contin. Dyn. Syst. Ser. B 1 (2001) 89-102.

[19] P.L. Lions, Mathematical Topics in Fluid Mechanics, Incompressible models, Vol. 1. Oxford University Press, UK (1996).

[20] F. Marche, Derivation of a new two-dimensional viscous shallow water model with varying topography, bottom friction and capillary effects. Eur. J. Mech. B, Fluids 26 (2007) 49-63.

[21] B. Mohammadi, O. Pironneau and F. Valentin, Rough boundaries and wall laws. Int. J. Numer. Methods Fluids 27 (1998) 169-177.

[22] O. Nwogu, Alternative form of Boussinesq equations for nearshore wave propagation. J. Waterw. Port Coast. Ocean Eng. ASCE 119 (1993) 618-638.

[23] D.H. Peregrine, Long waves on a beach. J. Fluid Mech. 27 (1967) 815-827. 
[24] B. Perthame, Kinetic formulation of conservation laws. Oxford University Press, UK (2002).

[25] B. Perthame and C. Simeoni, A kinetic scheme for the Saint-Venant system with a source term. Calcolo 38 (2001) $201-231$.

[26] M.J. Salençon and J.M. Thébault, Simulation model of a mesotrophic reservoir (Lac de Pareloup, France): Melodia, an ecosystem reservoir management model. Ecol. model. 84 (1996) 163-187.

[27] N.J. Shankar, H.F. Cheong and S. Sankaranarayanan, Multilevel finite-difference model for three-dimensional hydrodynamic circulation. Ocean Eng. 24 (1997) 785-816.

[28] F. Ursell, The long wave paradox in the theory of gravity waves. Proc. Cambridge Phil. Soc. 49 (1953) 685-694.

[29] M.A. Walkley, A numerical Method for Extended Boussinesq Shallow-Water Wave Equations. Ph.D. Thesis, University of Leeds, UK (1999). 